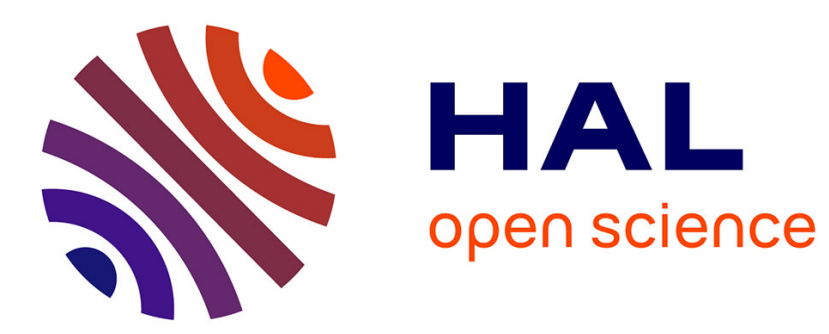

\title{
Form factor of any polyhedron: a general compact formula and its singularities
}

\author{
Bernard Croset
}

\section{To cite this version:}

Bernard Croset. Form factor of any polyhedron: a general compact formula and its singularities. Journal of Applied Crystallography, 2017, 50 (5), pp.1245-1255. 10.1107/S1600576717010147 . hal01610100

\section{HAL Id: hal-01610100 https://hal.science/hal-01610100}

Submitted on 5 Jul 2018

HAL is a multi-disciplinary open access archive for the deposit and dissemination of scientific research documents, whether they are published or not. The documents may come from teaching and research institutions in France or abroad, or from public or private research centers.
L'archive ouverte pluridisciplinaire HAL, est destinée au dépôt et à la diffusion de documents scientifiques de niveau recherche, publiés ou non, émanant des établissements d'enseignement et de recherche français ou étrangers, des laboratoires publics ou privés. 


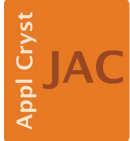

JOURNAL OF APPLIED CRYSTALLOGRAPHY

ISSN 1600-5767

Received 21 April 2017

Accepted 7 July 2017

Edited by V. T. Forsyth, Institut Laue-Langevin, France, and Keele University, UK

Keywords: X-ray diffraction; form factors; polyhedra; nanoparticles.

Supporting information: this article has supporting information at journals.iucr.org/j

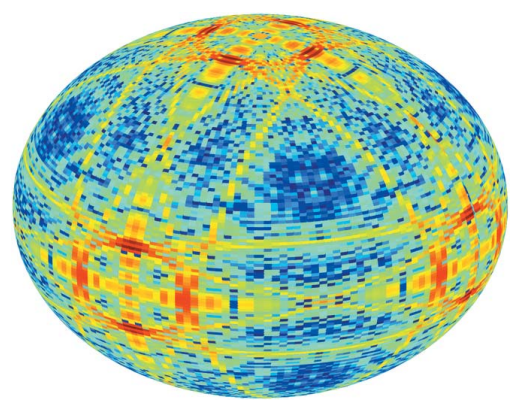

(C) 2017 International Union of Crystallography

\section{Form factor of any polyhedron: a general compact formula and its singularities}

\author{
Bernard Croset*
}

Sorbonne Universités, UPMC Univ Paris 06, CNRS-UMR 7588, Institut des NanoSciences de Paris, F-75005, Paris, France. *Correspondence e-mail: bernard.croset@insp.jussieu.fr

A general and compact formula is established for the form factor of any polyhedron, which involves only the apex coordinates and the apex connections. For large diffusion vector $\mathbf{q}$, the form factor behaves like $q^{-3}$ for generic directions, but it exhibits $q^{-2}$ singularities in the directions perpendicular to the edges and $q^{-1}$ singularities in the directions normal to the faces. General results are established for these singularities. Using a Python implementation, illustrative examples are discussed. The generality of the formula and of its singularities are likely to be important for any discussion of scattering from polyhedral particles.

\section{Introduction}

The increasing development of nanoscience has lead to an unprecedented control of nanoparticle synthesis (Goubet et al., 2013; Murphy et al., 2005; Xia et al., 2009; Xie et al., 2012; Zhang et al., 2012). The control of naonparticle shapes is of major importance for their physical properties in diverse fields: magnetism (Gruner et al., 2008), optics and plasmonics (Langille et al., 2012), and catalysis, for which the nature of the faces is crucial (Balmes et al., 2016; Yamada et al., 2011). Fine control of the shape and the size of nanoparticles has opened the route to the elaboration via self-assembly of superlattices of such nanoparticles (Cho et al., 2005; Geuchies et al., 2016; Goubet et al., 2016; Jones et al., 2010; Kalesaki et al., 2014; Lee et al., 2011).

X-ray scattering techniques are an important tool for studying and following these self-assembled nanoparticle system (Geuchies et al., 2016; Goubet et al., 2016; Jones et al., 2010; Renaud et al., 2009). As pointed out by Senesi and Lee, the calculation of the form factor of the particles is of major importance in the context of any scattering technique. On the one hand, the use of Green's theorem and surface triangulation approaches (Georg \& Tausch, 1994) leads to very efficient numerical methods for particles having 'smooth' surfaces (Chourou et al., 2013). On the other hand, for elementary polyhedra, the existence of very simple analytical formulae for the form factor indicates the presence of singularities for special directions of the diffusion vector $\mathbf{q}$. The existence of such singularities gives rise to a number of questions: Do these singularities exist for any polyhedron? What are their common properties? Analytically answering these questions is the subject of this paper.

Until now, two approaches have been employed to calculate analytically the form factor of specific polyhedra: (i) direct calculation of the integral on the polyhedron, which is the case for simple pyramids (tetrahedron, square pyramid etc.) or simple prisms (cube, parallelepiped etc.); (ii) cutting the polyhedron into elementary parts which are generally polygonal 
pyramids (Senesi \& Lee, 2015). These methods allow us to establish interesting results, but they lead to the construction of form factor catalogues (Babonneau, 2010; Lazzari, 2002). By formulating the calculation with simple vectorial operations, we will establish a general, compact and exact formula. This formula is valid for a very large class of polyhedra, those polyhedra severable into convex polyhedra, and it involves only the apex coordinates and the description of the apex connections. Its simplicity and generality allow one to identify the directions of the diffusion vector for which singularities appear. The first kind of singularity leads to an excess of diffusion in planes perpendicular to the edges, whereas the second kind leads to an excess of diffusion along lines normal to the faces. While the second singularity was already well known for each specific polyhedron, little attention has been paid so far to the first. Finally, we give a Python implementation of the formula and apply it to some illustrative cases.

\section{Form factor of any polygon}

\subsection{Form factor of a triangle}

Because of its importance in this work, we will establish in detail this well known result, allowing us to present our notations. Let us consider the triangle represented in Fig. 1, of apices $A_{1}, A_{2}, A_{3}$, the direction angles of the sides $A_{2} A_{3}, A_{1} A_{3}$, $A_{1} A_{2}$ being $\alpha_{1}, \alpha_{2}, \alpha_{3}$, respectively. We intend to calculate the form factor, i.e. the integral of $\exp (-i \mathbf{q} \cdot \mathbf{r})$ over the triangle surface, $F_{\Delta}=\iint_{\Delta} \exp (-i \mathbf{q} \cdot \mathbf{r}) \mathrm{d}^{2} s$. We have

$$
\begin{aligned}
& F_{\Delta}= \int_{x_{1}}^{x_{3}} \exp \left(-i q_{x} x\right)\left[\int_{y_{1}-\left(x-x_{1}\right) \cot \left(\alpha_{3}\right)}^{y_{1}-\left(x-x_{1}\right) \cot \left(\alpha_{2}\right)} \exp \left(-i q_{y} y\right) \mathrm{d} y\right] \mathrm{d} x \\
&+\int_{x_{3}}^{x_{2}} \exp \left(-i q_{x} x\right)\left[\int_{y_{1}-\left(x-x_{1}\right) \cot \left(\alpha_{3}\right)}^{y_{3}-\left(x-x_{3}\right) \cot \left(\alpha_{1}\right)} \exp \left(-i q_{y} y\right) \mathrm{d} y\right] \mathrm{d} x \\
&= \frac{i}{q_{y}} \int_{x_{1}}^{x_{2}} \exp \left(-i q_{x} x\right) \exp \left\{-i q_{y}\left[y_{1}-\left(x-x_{1}\right) \cot \left(\alpha_{3}\right)\right]\right\} \mathrm{d} x \\
&-\frac{i}{q_{y}} \int_{x_{1}}^{x_{3}} \exp \left(-i q_{x} x\right) \exp \left\{-i q_{y}\left[y_{1}-\left(x-x_{1}\right) \cot \left(\alpha_{2}\right)\right]\right\} \mathrm{d} x \\
&+\frac{i}{q_{y}} \int_{x_{2}}^{x_{3}} \exp \left(-i q_{x} x\right) \exp \left\{-i q_{y}\left[y_{2}-\left(x-x_{2}\right) \cot \left(\alpha_{1}\right)\right]\right\} \mathrm{d} x \\
&= \frac{-1}{q_{y}\left[q_{x}-q_{y} \cot \left(\alpha_{3}\right)\right]} \\
& \times\left\{\exp \left[-i\left(q_{x} x_{2}+q_{y} y_{2}\right)\right]-\exp \left[-i\left(q_{x} x_{1}+q_{y} y_{1}\right)\right]\right\} \\
&-\frac{-1}{q_{y}\left[q_{x}-q_{y} \cot \left(\alpha_{2}\right)\right]} \\
& \times\left\{\exp \left[-i\left(q_{x} x_{3}+q_{y} y_{3}\right)\right]-\exp \left[-i\left(q_{x} x_{1}+q_{y} y_{1}\right)\right]\right\} \\
&+\frac{-1}{q_{y}\left[q_{x}-q_{y} \cot \left(\alpha_{1}\right)\right]} \\
& \times\left\{\exp \left[-i\left(q_{x} x_{2}+q_{y} y_{2}\right)\right]-\exp \left[-i\left(q_{x} x_{3}+q_{y} y_{3}\right)\right]\right\} \\
&
\end{aligned}
$$

Three phases appear in this result: $q_{x} x_{1}+q_{y} y_{1}, q_{x} x_{2}+q_{y} y_{2}$ and $q_{x} x_{3}+q_{y} y_{3}$, with coefficients

$$
\begin{aligned}
& \frac{-1}{q_{y}\left[q_{x}-q_{y} \cot \left(\alpha_{2}\right)\right]}-\frac{-1}{q_{y}\left[q_{x}-q_{y} \cot \left(\alpha_{3}\right)\right]} \\
& =\frac{\sin \left(\alpha_{2}-\alpha_{3}\right)}{\left[q_{x} \sin \left(\alpha_{2}\right)-q_{y} \cos \left(\alpha_{2}\right)\right]\left[q_{x} \sin \left(\alpha_{3}\right)-q_{y} \cos \left(\alpha_{3}\right)\right]}, \\
& \frac{-1}{q_{y}\left[q_{x}-q_{y} \cot \left(\alpha_{3}\right)\right]}-\frac{-1}{q_{y}\left[q_{x}-q_{y} \cot \left(\alpha_{1}\right)\right]} \\
& =\frac{\sin \left(\alpha_{3}-\alpha_{1}\right)}{\left[q_{x} \sin \left(\alpha_{3}\right)-q_{y} \cos \left(\alpha_{3}\right)\right]\left[q_{x} \sin \left(\alpha_{1}\right)-q_{y} \cos \left(\alpha_{1}\right)\right]}, \quad \text { and } \\
& \frac{-1}{q_{y}\left[q_{x}-q_{y} \cot \left(\alpha_{1}\right)\right]}-\frac{-1}{q_{y}\left[q_{x}-q_{y} \cot \left(\alpha_{2}\right)\right]} \\
& =\frac{\sin \left(\alpha_{1}-\alpha_{2}\right)}{\left[q_{x} \sin \left(\alpha_{1}\right)-q_{y} \cos \left(\alpha_{1}\right)\right]\left[q_{x} \sin \left(\alpha_{2}\right)-q_{y} \cos \left(\alpha_{2}\right)\right]} .
\end{aligned}
$$

A major point in the whole following calculation is to note that operations on vectors such as the scalar product, $\mathbf{V} \cdot \mathbf{W}$, the vector product, $\mathbf{V} \times \mathbf{W}$, and the mixed product, $\mathbf{U} \cdot(\mathbf{V} \times \mathbf{W})=(\mathbf{U}, \mathbf{V}, \mathbf{W})$, are invariant under changes of basis and rotations. Therefore, we will search for a formulation of the form factor using such vectorial operations. Denoting by n the unit vector normal to the $A_{1} A_{2} A_{3}$ plane which completes the right-handed axis system, the coefficient of the phase term $q_{x} x_{1}+q_{y} y_{1}$ can be rewritten as $\left[\mathbf{n} \cdot\left(\mathbf{A}_{2} \mathbf{A}_{1} \times \mathbf{A}_{1} \mathbf{A}_{3}\right)\right] /$ $\left[\left(\mathbf{q} \cdot \mathbf{A}_{2} \mathbf{A}_{1}\right)\left(\mathbf{q} \cdot \mathbf{A}_{1} \mathbf{A}_{3}\right)\right]$ and we have finally for the form factor

$$
F_{\Delta}=\sum_{i=1}^{3} \exp \left(-i \mathbf{q} \cdot \mathbf{O} \mathbf{A}_{i}\right) \frac{\mathbf{n} \cdot\left(\mathbf{A}_{i+1} \mathbf{A}_{i} \times \mathbf{A}_{i} \mathbf{A}_{i-1}\right)}{\left(\mathbf{q} \cdot \mathbf{A}_{i+1} \mathbf{A}_{i}\right)\left(\mathbf{q} \cdot \mathbf{A}_{i} \mathbf{A}_{i-1}\right)} .
$$

In this equation, the operations on the indexes must be done modulo 3. This will be a general rule of great importance in the whole paper: for index $i$, varying in the range $1 \ldots p$, the operation $i+k$ or $i-k$ must be understood modulo $p$.

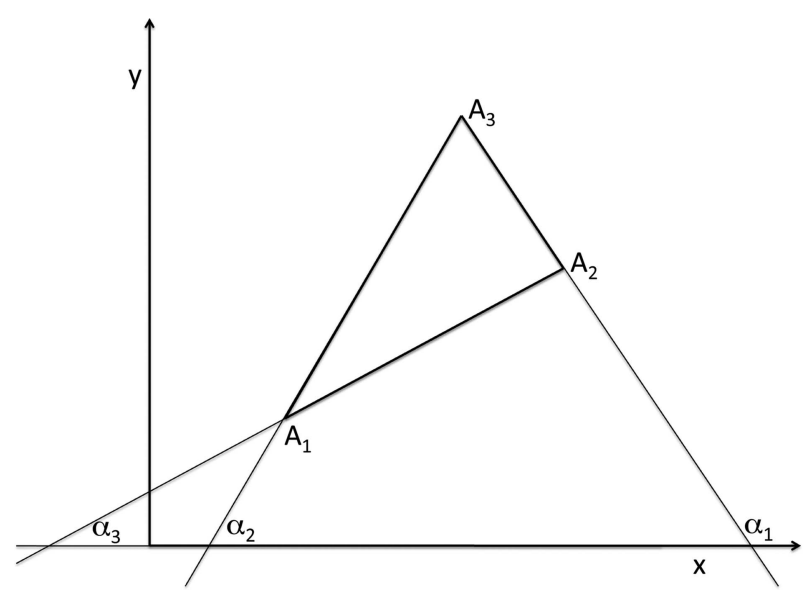

Figure 1

Scheme of the triangle $A_{1} A_{2} A_{3}$. 


\subsection{Form factor of simple polygons}

We show by induction in Appendix $A$ that the form factor of a simple $p$-gon, i.e. a simple uncrossed polygon with $p$ apices, can be written as

$$
F_{\mathrm{plgn}}=\sum_{i=1}^{p} \exp \left(-i \mathbf{q} \cdot \mathbf{O} \mathbf{A}_{i}\right) \frac{\mathbf{n} \cdot\left(\mathbf{A}_{i+1} \mathbf{A}_{i} \times \mathbf{A}_{i} \mathbf{A}_{i-1}\right)}{\left(\mathbf{q} \cdot \mathbf{A}_{i+1} \mathbf{A}_{i}\right)\left(\mathbf{q} \cdot \mathbf{A}_{i} \mathbf{A}_{i-1}\right)}
$$

This formula can be obtained by direct calculation following the calculation scheme employed for the triangle, but its demonstration by induction illustrates a cutting procedure quite useful in the context of this article.

\section{Form factor of a polyhedron}

\subsection{Form factor of a tetrahedron}

Let us consider the tetrahedron represented in Fig. 2, of base $A_{1} A_{2} A_{3}$ parallel to the $x y$ plane at height $z_{\mathrm{b}}$, the fourth apex being $A_{0}$ at height $z_{0}$.

The form factor is given by

$$
\begin{aligned}
F_{\text {tetra }}= & \iiint \exp (-i \mathbf{q} \cdot \mathbf{r}) \mathrm{d}^{3} v \\
= & \exp \left(-i \mathbf{q} \cdot \mathbf{O} \mathbf{A}_{0}\right) \\
& \times \int_{z_{0}}^{z_{\mathrm{b}}} \sum_{i=1}^{3} \frac{\mathbf{n} \cdot\left(\mathbf{a}_{i+1} \mathbf{a}_{i} \times \mathbf{a}_{i-1} \mathbf{a}_{i}\right)}{\left(\mathbf{q} \cdot \mathbf{a}_{i+1} \mathbf{a}_{i}\right)\left(\mathbf{q} \cdot \mathbf{a}_{i-1} \mathbf{a}_{i}\right)} \exp \left(-i \mathbf{q} \cdot \mathbf{A}_{0} \mathbf{a}_{i}\right) \mathrm{d} z .
\end{aligned}
$$

Since the triangles $A_{1} A_{2} A_{3}$ and $a_{1} a_{2} a_{3}$ are similar in a homothetic transformation of centre $A_{0}$ and ratio $\left(z-z_{0}\right) /\left(z_{\mathrm{b}}-z_{0}\right)$, we have

$$
\frac{\mathbf{n} \cdot\left(\mathbf{a}_{i+1} \mathbf{a}_{i} \times \mathbf{a}_{i-1} \mathbf{a}_{i}\right)}{\left(\mathbf{q} \cdot \mathbf{a}_{i+1} \mathbf{a}_{i}\right)\left(\mathbf{q} \cdot \mathbf{a}_{i-1} \mathbf{a}_{i}\right)}=\frac{\mathbf{n} \cdot\left(\mathbf{A}_{i+1} \mathbf{A}_{i} \times \mathbf{A}_{i-1} \mathbf{A}_{i}\right)}{\left(\mathbf{q} \cdot \mathbf{A}_{i+1} \mathbf{A}_{i}\right)\left(\mathbf{q} \cdot \mathbf{A}_{i-1} \mathbf{A}_{i}\right)}
$$

and $\mathbf{A}_{0} \mathbf{a}_{i}=\mathbf{A}_{0} \mathbf{A}_{i}\left(z-z_{0}\right) /\left(z_{\mathrm{b}}-z_{0}\right)$. Therefore, we obtain

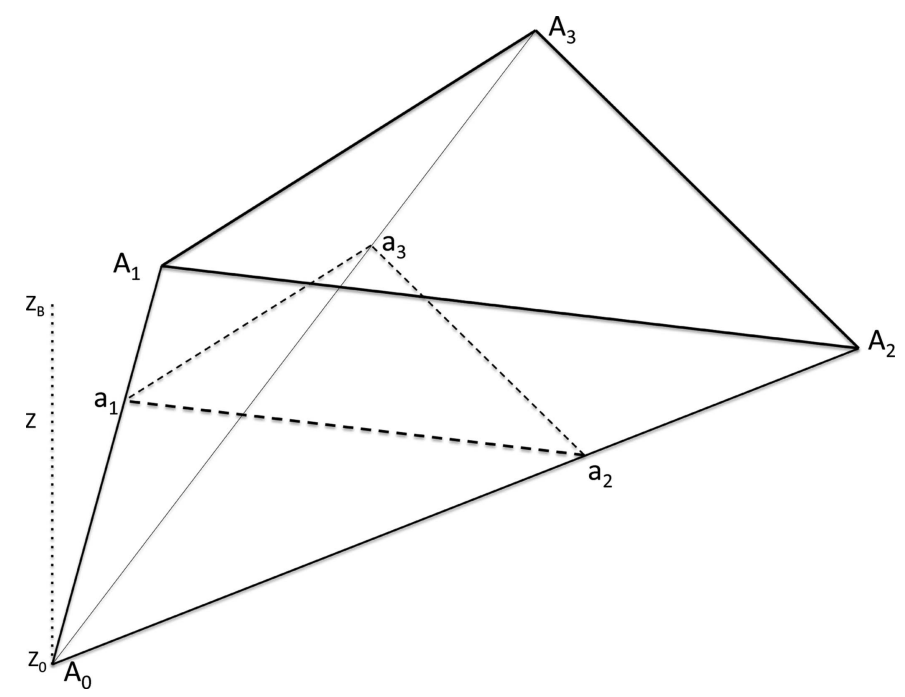

Figure 2

Tetrahedron. The two triangles marked with the bold and dashed lines are similar in a homothetic transformation of centre $A_{0}$ and ratio $\left(z-z_{0}\right) /\left(z_{\mathrm{b}}-z_{0}\right)$

$$
\begin{aligned}
F_{\text {tetra }}= & \exp \left(-i \mathbf{q} \cdot \mathbf{O} \mathbf{A}_{0}\right) \int_{z_{0}}^{z_{\mathrm{b}}} \sum_{i=1}^{3} \frac{\mathbf{n} \cdot\left(\mathbf{A}_{i+1} \mathbf{A}_{i} \times \mathbf{A}_{i-1} \mathbf{A}_{i}\right)}{\left(\mathbf{q} \cdot \mathbf{A}_{i+1} \mathbf{A}_{i}\right)\left(\mathbf{q} \cdot \mathbf{A}_{i-1} \mathbf{A}_{i}\right)} \\
& \times \exp \left(-i \mathbf{q} \cdot \mathbf{A}_{0} \mathbf{A}_{i} \frac{z-z_{0}}{z_{\mathrm{b}}-z_{0}}\right) \mathrm{d} z
\end{aligned}
$$

Note that it is not necessary for $A_{0}$ to be vertically aligned with the triangle barycentre to obtain such an integral, as assumed by Senesi \& Lee (2015). After integration, one obtains

$$
\begin{aligned}
F_{\text {tetra }}= & \left(z_{\mathrm{b}}-z_{0}\right) \exp \left(-i \mathbf{q} \cdot \mathbf{O} \mathbf{A}_{0}\right) \\
& \times \sum_{i=1}^{3}\left[\frac{\mathbf{n} \cdot\left(\mathbf{A}_{i+1} \mathbf{A}_{i} \times \mathbf{A}_{i-1} \mathbf{A}_{i}\right)}{\left(\mathbf{q} \cdot \mathbf{A}_{i+1} \mathbf{A}_{i}\right)\left(\mathbf{q} \cdot \mathbf{A}_{i-1} \mathbf{A}_{i}\right)} \frac{\exp \left(-i \mathbf{q} \cdot \mathbf{A}_{0} \mathbf{A}_{i}\right)+1}{-i\left(\mathbf{q} \cdot \mathbf{A}_{0} \mathbf{A}_{i}\right)}\right] \\
= & i \sum_{i=1}^{3}\left[\frac{\mathbf{A}_{i} \mathbf{A}_{0} \cdot\left(\mathbf{A}_{i+1} \mathbf{A}_{i} \times \mathbf{A}_{i-1} \mathbf{A}_{i}\right)}{\left(\mathbf{q} \cdot \mathbf{A}_{i+1} \mathbf{A}_{i}\right)\left(\mathbf{q} \cdot \mathbf{A}_{i-1} \mathbf{A}_{i}\right)\left(\mathbf{q} \cdot \mathbf{A}_{0} \mathbf{A}_{i}\right)}\right. \\
& \left.\times \exp \left(-i \mathbf{q} \cdot \mathbf{O} \mathbf{A}_{i}\right)\right]-i \exp \left(-i \mathbf{q} \cdot \mathbf{O} \mathbf{A}_{0}\right) \\
& \times \sum_{i=1}^{3} \frac{\mathbf{A}_{i} \mathbf{A}_{0} \cdot\left(\mathbf{A}_{i+1} \mathbf{A}_{i} \times \mathbf{A}_{i-1} \mathbf{A}_{i}\right)}{\left(\mathbf{q} \cdot \mathbf{A}_{i+1} \mathbf{A}_{i}\right)\left(\mathbf{q} \cdot \mathbf{A}_{i-1} \mathbf{A}_{i}\right)\left(\mathbf{q} \cdot \mathbf{A}_{0} \mathbf{A}_{i}\right)}
\end{aligned}
$$

where the operations on the indexes are done modulo 3 in the set $1,2,3$.

\subsection{Form factor of a polygonal pyramid}

Using the same procedure of calculation, the previous formula can be generalized to the form factor of a polygonal pyramid:

$$
\begin{aligned}
F_{\mathrm{pyr}}= & i \sum_{i=1}^{p}\left[\frac{\mathbf{A}_{0} \mathbf{A}_{i} \cdot\left(\mathbf{A}_{i+1} \mathbf{A}_{i} \times \mathbf{A}_{i-1} \mathbf{A}_{i}\right)}{\left(\mathbf{q} \cdot \mathbf{A}_{i+1} \mathbf{A}_{i}\right)\left(\mathbf{q} \cdot \mathbf{A}_{i-1} \mathbf{A}_{i}\right)\left(\mathbf{q} \cdot \mathbf{A}_{0} \mathbf{A}_{i}\right)} \exp \left(-i \mathbf{q} \cdot \mathbf{O} \mathbf{A}_{i}\right)\right] \\
& -i \exp \left(-i \mathbf{q} \cdot \mathbf{O} \mathbf{A}_{0}\right) \\
& \times \sum_{i=1}^{p}\left[\frac{\mathbf{A}_{0} \mathbf{A}_{i} \cdot\left(\mathbf{A}_{i+1} \mathbf{A}_{i} \times \mathbf{A}_{i-1} \mathbf{A}_{i}\right)}{\left(\mathbf{q} \cdot \mathbf{A}_{i+1} \mathbf{A}_{i}\right)\left(\mathbf{q} \cdot \mathbf{A}_{i-1} \mathbf{A}_{i}\right)\left(\mathbf{q} \cdot \mathbf{A}_{0} \mathbf{A}_{i}\right)}\right]
\end{aligned}
$$

This result is essential. It will allow us to calculate the general formula for simple polyhedra.

\subsection{Compact formula for the form factor of a simple polyhedron}

We will proceed in two steps: first we will establish a compact formula for a special class of polyhedra, the 'not too concave' polyhedra, and second we will demonstrate in appendices some lemmas which allow us to generalize the validity of that formula for a quite large class of polyhedra, the polyhedra severable into convex parts.

Let us consider a polyhedron for which there exists a finite locus where all the faces can be seen without passing through the polyhedron exterior. This class of polyhedra contains all the convex polyhedra but also all the polyhedra that are not too concave. Choosing a point $M$ in this locus, the polyhedron can be divided in polygonal pyramids. Labelling by $j$ the $n_{\mathrm{f}}$ faces and by $v_{j, k}$ the ordered circuit of the $m(j)$ apices of the $j$ th face, one obtains using formula (8) 


$$
\begin{aligned}
F_{\text {plhd }}= & i \sum_{j=1}^{n_{\mathrm{f}}}\left[\sum_{k=1}^{m(j)} \frac{\mathbf{M} \mathbf{A}_{v_{j, k}} \cdot\left(\mathbf{A}_{v_{j, k+1}} \mathbf{A}_{v_{j, k}} \times \mathbf{A}_{v_{j, k}} \mathbf{A}_{v_{j, k-1}}\right)}{\left(\mathbf{q} \cdot \mathbf{A}_{v_{j, k+1}} \mathbf{A}_{v_{j, k}}\right)\left(\mathbf{q} \cdot \mathbf{A}_{v_{j, k}} \mathbf{A}_{v_{j, k-1}}\right)\left(\mathbf{q} \cdot \mathbf{M} \mathbf{A}_{v_{j, k}}\right)}\right. \\
& \left.\times \exp \left(-i \mathbf{q} \cdot \mathbf{O} \mathbf{A}_{v_{j, k}}\right)\right]-i \exp (-i \mathbf{q} \cdot \mathbf{O M}) \\
& \times \sum_{j=1}^{n_{\mathrm{f}}}\left[\sum_{k=1}^{m(j)} \frac{\mathbf{M} \mathbf{A}_{v_{j, k}} \cdot\left(\mathbf{A}_{v_{j, k+1}} \mathbf{A}_{v_{j, k}} \times \mathbf{A}_{v_{j, k}} \mathbf{A}_{v_{j, k-1}}\right)}{\left(\mathbf{q} \cdot \mathbf{A}_{v_{j, k+1}} \mathbf{A}_{v_{j, k}}\right)\left(\mathbf{q} \cdot \mathbf{A}_{v_{j, k}} \mathbf{A}_{v_{j, k-1}}\right)\left(\mathbf{q} \cdot \mathbf{M} \mathbf{A}_{v_{j, k}}\right)}\right] .
\end{aligned}
$$

The phase term $\exp \left(-i \mathbf{q} \cdot \mathbf{O} \mathbf{A}_{i}\right)$ associated with the apex $A_{i}$ appears several times in formula (9). Let us label by $w_{i, s}$ the circuit of the $p_{i}$ apices connected to the apex $i$. The order of this connection circuit can be determined unambiguously by the order of the face circuits as shown in Fig. 3.

Using these indices, the summation may be rewritten as

$$
\begin{aligned}
F_{\text {plhd }}= & i \sum_{i=1}^{n_{\mathrm{a}}}\left[\sum_{s=1}^{p(i)} \frac{\mathbf{M} \mathbf{A}_{i} \cdot\left(\mathbf{A}_{w_{i, s}} \mathbf{A}_{i} \times \mathbf{A}_{i} \mathbf{A}_{w_{i, s-1}}\right)}{\left(\mathbf{q} \cdot \mathbf{A}_{w_{i, s}} \mathbf{A}_{i}\right)\left(\mathbf{q} \cdot \mathbf{A}_{i} \mathbf{A}_{w_{i, s-1}}\right)\left(\mathbf{q} \cdot \mathbf{M} \mathbf{A}_{i}\right)}\right] \\
& \times \exp \left(-i \mathbf{q} \cdot \mathbf{O} \mathbf{A}_{i}\right)-i \exp (-i \mathbf{q} \cdot \mathbf{O M}) \\
& \times \sum_{i=1}^{n_{\mathrm{a}}}\left[\sum_{s=1}^{p(i)} \frac{\mathbf{M A}_{i} \cdot\left(\mathbf{A}_{w_{i, s}} \mathbf{A}_{i} \times \mathbf{A}_{i} \mathbf{A}_{w_{i, s-1}}\right)}{\left(\mathbf{q} \cdot \mathbf{A}_{w_{i, s}} \mathbf{A}_{i}\right)\left(\mathbf{q} \cdot \mathbf{A}_{i} \mathbf{A}_{w_{i, s-1}}\right)\left(\mathbf{q} \cdot \mathbf{M} \mathbf{A}_{i}\right)}\right],
\end{aligned}
$$

where $n_{\mathrm{a}}$ is the number of apices. Note that the phase term associated with each apex is involved in the inner summation when using the faces' circuits while it is involved in the outer summation when using the apices' circuits.

The formula obtained may seem surprising because of its explicit dependence on the choice of $M$. This apparent dependency can be removed, which will lead to our general compact formula for the form factor. Let us start with the coefficient $G_{i}$ of the phase term $\exp \left(-i \mathbf{q} \cdot \mathbf{O \mathbf { A } _ { i }}\right)$.

$$
G_{i}=\sum_{s=1}^{p(i)} \frac{\mathbf{M} \mathbf{A}_{i} \cdot\left(\mathbf{A}_{w_{i, s}} \mathbf{A}_{i} \times \mathbf{A}_{i} \mathbf{A}_{w_{i, s-1}}\right)}{\left(\mathbf{q} \cdot \mathbf{A}_{w_{i, s}} \mathbf{A}_{i}\right)\left(\mathbf{q} \cdot \mathbf{A}_{i} \mathbf{A}_{w_{i, s-1}}\right)\left(\mathbf{q} \cdot \mathbf{M} \mathbf{A}_{i}\right)}=\frac{\mathbf{M} \mathbf{A}_{i} \cdot \mathbf{V}_{i}}{\mathbf{q} \cdot \mathbf{M} \mathbf{A}_{i}}
$$

with

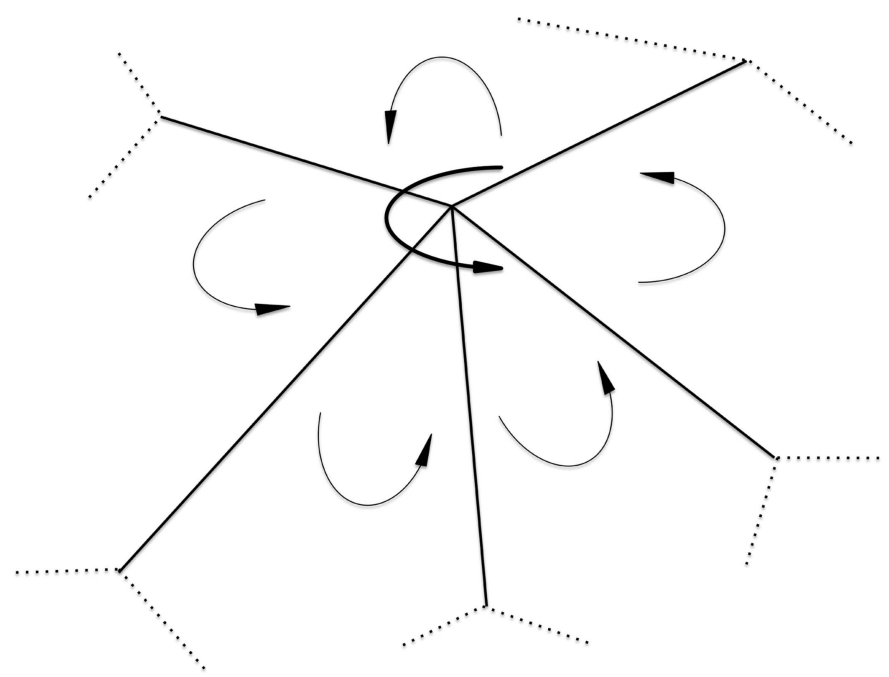

Figure 3

Principle of orientations of the edges' circuits.

$$
\mathbf{V}_{i}=\sum_{s=1}^{p(i)} \frac{\mathbf{A}_{w_{i, s}} \mathbf{A}_{i} \times \mathbf{A}_{i} \mathbf{A}_{w_{i, s-1}}}{\left(\mathbf{q} \cdot \mathbf{A}_{w_{i, s}} \mathbf{A}_{i}\right)\left(\mathbf{q} \cdot \mathbf{A}_{i} \mathbf{A}_{w_{i, s-1}}\right)}
$$

We will show that $\mathbf{V}_{i}$ is parallel to $\mathbf{q}$. For this purpose, we will calculate $\mathbf{q} \times \mathbf{V}_{i}$. We have

$$
\begin{aligned}
\mathbf{q} \times \mathbf{V}_{i} & =\sum_{s=1}^{p(i)}\left[\frac{\mathbf{q} \times\left(\mathbf{A}_{w_{i, s}} \mathbf{A}_{i} \times \mathbf{A}_{w_{i, s-1}} \mathbf{A}_{i}\right)}{\left(\mathbf{q} \cdot \mathbf{A}_{w_{i, s}} \mathbf{A}_{i}\right)\left(\mathbf{q} \cdot \mathbf{A}_{w_{i, s-1}} \mathbf{A}_{i}\right)}\right] \\
& =\sum_{s=1}^{p(i)}\left[\frac{\left(\mathbf{q} \cdot \mathbf{A}_{w_{i, s-1}} \mathbf{A}_{i}\right) \mathbf{A}_{w_{i, s}} \mathbf{A}_{i}-\left(\mathbf{q} \cdot \mathbf{A}_{w_{i, s}} \mathbf{A}_{i}\right) \mathbf{A}_{w_{i, s-1}} \mathbf{A}_{i}}{\left(\mathbf{q} \cdot \mathbf{A}_{w_{i, s}} \mathbf{A}_{i}\right)\left(\mathbf{q} \cdot \mathbf{A}_{w_{i, s-1}} \mathbf{A}_{i}\right)}\right] \\
& =\sum_{s=1}^{p(i)}\left[\frac{\mathbf{A}_{w_{i, s}} \mathbf{A}_{i}}{\left(\mathbf{q} \cdot \mathbf{A}_{w_{i, s}} \mathbf{A}_{i}\right)}-\frac{\mathbf{A}_{w_{i, s-1}} \mathbf{A}_{i}}{\left(\mathbf{q} \cdot \mathbf{A}_{w_{i, s-1}} \mathbf{A}_{i}\right)}\right] \\
& =\sum_{s=1}^{p(i)} \frac{\mathbf{A}_{w_{i, s}} \mathbf{A}_{i}}{\left(\mathbf{q} \cdot \mathbf{A}_{w_{i, s}} \mathbf{A}_{i}\right)}-\sum_{s=1}^{p(i)} \frac{\mathbf{A}_{w_{i, s-1}} \mathbf{A}_{i}}{\left(\mathbf{q} \cdot \mathbf{A}_{w_{i, s-1}} \mathbf{A}_{i}\right)} \\
& =\mathbf{0} .
\end{aligned}
$$

Therefore, we have $\mathbf{V}_{i}=t_{i} \mathbf{q}$ with $t_{i}=\left(\mathbf{V}_{i} \cdot \mathbf{q}\right) / q^{2}$. This leads to

$$
G_{i}=\frac{\mathbf{M} \mathbf{A}_{i} \cdot\left(t_{i} \mathbf{q}\right)}{\mathbf{q} \cdot \mathbf{M} \mathbf{A}_{i}}=t_{i}=\sum_{s=1}^{p(i)}\left[\frac{\mathbf{q} \cdot\left(\mathbf{A}_{w_{i, s}} \mathbf{A}_{i} \times \mathbf{A}_{w_{i, s-1}} \mathbf{A}_{i}\right)}{\left(\mathbf{q} \cdot \mathbf{A}_{w_{i, s}} \mathbf{A}_{i}\right)\left(\mathbf{q} \cdot \mathbf{A}_{w_{i, s-1}} \mathbf{A}_{i}\right) q^{2}}\right]
$$

which does not depend on $M$ and which can be calculated with only the apex coordinates. At this step, the remaining dependence of the form factor on $M$ is concentrated in a term where the phase $\mathbf{q} \cdot \mathbf{O M}$ appears with a rational function of the $M$ coordinates. Since the form factor must not depend on $M$, this rational fraction must be zero. A direct geometrical proof of this vanishing can be found in Appendix B. Finally, we have

$F_{\text {plhd }}=i \sum_{i=1}^{n_{\mathrm{a}}}\left[\sum_{s=1}^{p(i)} \frac{\mathbf{q} \cdot\left(\mathbf{A}_{w_{i, s}} \mathbf{A}_{i} \times \mathbf{A}_{w_{i, s-1}} \mathbf{A}_{i}\right)}{\left(\mathbf{q} \cdot \mathbf{A}_{w_{i, s}} \mathbf{A}_{i}\right)\left(\mathbf{q} \cdot \mathbf{A}_{w_{i, s-1}} \mathbf{A}_{i}\right) q^{2}}\right] \exp \left(-i \mathbf{q} \cdot \mathbf{O} \mathbf{A}_{i}\right)$.

The following points must be noted:

(1) The expression of the form factor involves only the apex coordinates and the topology of their connections but, in its final form, no particular cutting of the polyhedron is involved.

(2) For large $q$ values, the form factor behaves like $q^{-3}$.

(3) For the purpose of demonstration, we have used a special cutting and we have restrained our discussion to not too concave polyhedra, i.e. polyhedra for which there exists a finite locus where all the faces can be seen without passing through the polyhedron exterior. We give in Appendix $C$ a method which allows one to generalize the validity of this formula in its same form to any polyhedron severable into convex parts. While a little more technical, this generalization is very similar to the inductive demonstration given previously for the form factor of a $p$-gon.

\section{The different singularities}

In the denominator of formula (15) which leads to the form factor, the diffusion vector $\mathbf{q}$ is involved in several scalar products. This clearly shows that there exist directions of $\mathbf{q}$ for 
which the form factor presents singularities: $\mathbf{q}$ may be perpendicular to a single edge; $\mathbf{q}$ may be perpendicular to two consecutive edges in the circuit around an apex, in which case $\mathbf{q}$ is normal to the face to which these two edges belong; or $\mathbf{q}$ may be zero, leading to a zone centre singularity.

\subsection{First-kind singularities: q perpendicular to an edge}

Let us study the singularity which occurs when $\mathbf{q}$ is perpendicular to the edge $\mathbf{A}_{i} \mathbf{A}_{j}$ without being normal to either of the two faces to which this edge belongs. Defining $s$ and $t$ by the equalities $w_{i, s}=j$ and $w_{j, t}=i$, we will set $j_{-}=w_{i, s-1}$, $j_{+}=w_{i, s+1}, i_{-}=w_{j, t-1}$ and $i_{+}=w_{j, t+1}$. The four terms involving the scalar product $\mathbf{q} \cdot \mathbf{A}_{i} \mathbf{A}_{j}$ are written as

$$
\begin{aligned}
{\left[\frac{\mathbf{q} \cdot\left(\mathbf{A}_{j+} \mathbf{A}_{i} \times \mathbf{A}_{j} \mathbf{A}_{i}\right)}{\left(\mathbf{q} \cdot \mathbf{A}_{j} \mathbf{A}_{i}\right)\left(\mathbf{q} \cdot \mathbf{A}_{j+} \mathbf{A}_{i}\right) q^{2}}\right] \exp \left(-i \mathbf{q} \cdot \mathbf{O} \mathbf{A}_{i}\right) } & =\frac{C_{i}}{q^{2}\left(\mathbf{q} \cdot \mathbf{A}_{j} \mathbf{A}_{i}\right)} \\
& \times \exp \left(-i \mathbf{q} \cdot \mathbf{O} \mathbf{A}_{i}\right), \\
{\left[\frac{\mathbf{q} \cdot\left(\mathbf{A}_{j} \mathbf{A}_{i} \times \mathbf{A}_{j-} \mathbf{A}_{i}\right)}{\left(\mathbf{q} \cdot \mathbf{A}_{j} \mathbf{A}_{i}\right)\left(\mathbf{q} \cdot \mathbf{A}_{j-} \mathbf{A}_{i}\right) q^{2}}\right] \exp \left(-i \mathbf{q} \cdot \mathbf{O} \mathbf{A}_{i}\right) } & =\frac{D_{i}}{q^{2}\left(\mathbf{q} \cdot \mathbf{A}_{j} \mathbf{A}_{i}\right)} \\
& \times \exp \left(-i \mathbf{q} \cdot \mathbf{O} \mathbf{A}_{i}\right), \\
{\left[\frac{\mathbf{q} \cdot\left(\mathbf{A}_{i+} \mathbf{A}_{j} \times \mathbf{A}_{i} \mathbf{A}_{j}\right)}{\left(\mathbf{q} \cdot \mathbf{A}_{i} \mathbf{A}_{j}\right)\left(\mathbf{q} \cdot \mathbf{A}_{i+} \mathbf{A}_{j}\right) q^{2}}\right] \exp \left(-i \mathbf{q} \cdot \mathbf{O} \mathbf{A}_{j}\right) } & =\frac{C_{j}}{q^{2}\left(\mathbf{q} \cdot \mathbf{A}_{i} \mathbf{A}_{j}\right)} \\
& \times \exp \left(-i \mathbf{q} \cdot \mathbf{O} \mathbf{A}_{j}\right), \\
{\left[\frac{\mathbf{q} \cdot\left(\mathbf{A}_{i} \mathbf{A}_{j} \times \mathbf{A}_{i-} \mathbf{A}_{j}\right)}{\left(\mathbf{q} \cdot \mathbf{A}_{i} \mathbf{A}_{j}\right)\left(\mathbf{q} \cdot \mathbf{A}_{i-} \mathbf{A}_{j}\right) q^{2}}\right] \exp \left(-i \mathbf{q} \cdot \mathbf{O} \mathbf{A}_{j}\right) } & =\frac{D_{j}}{q^{2}\left(\mathbf{q} \cdot \mathbf{A}_{i} \mathbf{A}_{j}\right)} \\
& \times \exp \left(-i \mathbf{q} \cdot \mathbf{O} \mathbf{A}_{j}\right),
\end{aligned}
$$

and their sum is written as

$$
\begin{aligned}
\frac{C_{i}+D_{i}}{q^{2}\left(\mathbf{q} \cdot \mathbf{A}_{j} \mathbf{A}_{i}\right)} \exp \left(-i \mathbf{q} \cdot \mathbf{O} \mathbf{A}_{i}\right)-\frac{C_{j}+D_{j}}{q^{2}\left(\mathbf{q} \cdot \mathbf{A}_{j} \mathbf{A}_{i}\right)} \exp \left(-i \mathbf{q} \cdot \mathbf{O} \mathbf{A}_{j}\right) \\
=\left\{\frac{C_{i}+D_{i}}{q^{2}\left(\mathbf{q} \cdot \mathbf{A}_{j} \mathbf{A}_{i}\right)}\left[\exp \left(-i \mathbf{q} \cdot \mathbf{A}_{j} \mathbf{A}_{i}\right)-1\right]\right. \\
\left.+\frac{C_{i}+D_{i}-C_{j}-D_{j}}{q^{2}\left(\mathbf{q} \cdot \mathbf{A}_{j} \mathbf{A}_{i}\right)}\right\} \exp \left(-i \mathbf{q} \cdot \mathbf{O} \mathbf{A}_{j}\right) .
\end{aligned}
$$

We will denote by $\mathbf{n}_{-}$the unit normal to the face to which $A_{i_{+}}$, $A_{j}, A_{i}, A_{j_{-}}$belong and by $\mathbf{n}_{+}$the unit normal to the face to which $A_{i_{-}}, A_{j}, A_{i}, A_{j_{+}}$belong. Writing $\mathbf{q}=\alpha \mathbf{n}_{+}+\beta \mathbf{n}_{-}+$ $\gamma\left(\mathbf{n}_{-} \times \mathbf{n}_{+}\right)$and $\mathbf{A}_{i} \mathbf{A}_{j}=a\left(\mathbf{n}_{-} \times \mathbf{n}_{+}\right)$, we obtain

$$
C_{i 0}=\lim _{\left(\mathbf{q} \cdot \mathbf{A}_{i} \mathbf{A}_{j}\right) \rightarrow 0} C_{i}=\lim _{\gamma \rightarrow 0} C_{i}=\frac{a}{\beta}\left[\alpha+\beta\left(\mathbf{n}_{+} \cdot \mathbf{n}_{-}\right)\right] .
$$

The key point to note is that this limit is independent of $A_{j}$. Thus, we have $C_{i 0}=D_{j 0}=(a / \beta)\left[\alpha+\beta\left(\mathbf{n}_{+} \cdot \mathbf{n}_{-}\right)\right]$and $D_{i 0}=C_{j 0}=(a / \alpha)\left[\beta+\alpha\left(\mathbf{n}_{+} \cdot \mathbf{n}_{-}\right)\right]$. A series expansion leads for the whole singularity to

$$
\begin{aligned}
& a\left\{\left[\frac{\alpha}{\beta}+\frac{\beta}{\alpha}+2\left(\mathbf{n}_{+} \cdot \mathbf{n}_{-}\right)\right] \frac{\exp \left(-i \mathbf{q} \cdot \mathbf{A}_{j} \mathbf{A}_{i}\right)-1}{q^{2}\left(\mathbf{q} \cdot \mathbf{A}_{j} \mathbf{A}_{i}\right)}+\frac{E}{q^{2}}\right\} \\
& \quad \times \exp \left(-i \mathbf{q} \cdot \mathbf{O} \mathbf{A}_{j}\right) \\
&=a\left\{\left[\frac{\left(\mathbf{q} \cdot \mathbf{n}_{+}\right)-\left(\mathbf{q} \cdot \mathbf{n}_{-}\right)\left(\mathbf{n}_{-} \cdot \mathbf{n}_{+}\right)}{\left(\mathbf{q} \cdot \mathbf{n}_{-}\right)-\left(\mathbf{q} \cdot \mathbf{n}_{+}\right)\left(\mathbf{n}_{-} \cdot \mathbf{n}_{+}\right)}\right.\right. \\
&\left.\quad+\frac{\left(\mathbf{q} \cdot \mathbf{n}_{-}\right)-\left(\mathbf{q} \cdot \mathbf{n}_{+}\right)\left(\mathbf{n}_{-} \cdot \mathbf{n}_{+}\right)}{\left(\mathbf{q} \cdot \mathbf{n}_{+}\right)-\left(\mathbf{q} \cdot \mathbf{n}_{-}\right)\left(\mathbf{n}_{-} \cdot \mathbf{n}_{+}\right)}+2\left(\mathbf{n}_{+} \cdot \mathbf{n}_{-}\right)\right] \\
&\left.\times \frac{\exp \left(-i \mathbf{q} \cdot \mathbf{A}_{j} \mathbf{A}_{i}\right)-1}{q^{2}\left(\mathbf{q} \cdot \mathbf{A}_{j} \mathbf{A}_{i}\right)}+\frac{E}{q^{2}}\right\} \exp \left(-i \mathbf{q} \cdot \mathbf{O} \mathbf{A}_{j}\right),
\end{aligned}
$$

where $E$ is obtained by a series expansion of $C_{i}$ and $D_{j}$ to the first order. The singularity is the sum of a cardinal sine term and a diffuse term. The following points must be noted:

(a) The locus of the first-kind singularity is a plane normal to the edge and intersecting the origin, leading to great circles on a sphere of radius $q$.

(b) For large $q$ values, the first-kind singularity behaves like $q^{-2}$.

(c) Through $a$, the first-kind singularity is proportional to the edge length, $A_{i} A_{j}$.

(d) The first-kind singularity exhibits fringes of period $2 \pi / A_{i} A_{j}$ in the direction of the edge.

\subsection{Second-kind singularities: q normal to a face}

Let us study the singularity which occurs when $\mathbf{q}$ is normal to a face without being zero. We denote by $\mathbf{n}$ the unit normal to this face pointing toward the exterior. We have $\mathbf{q}=q_{\perp} \mathbf{n}+\mathbf{q}_{\|}$. We will index by $j$ the $m_{\mathrm{f}}$ apices of this face. For each of these apices, there is one term in the inner sum for which the two scalar products of the denominator are zero when $\mathbf{q}_{\|}=\mathbf{0}$. Grouping all these singular terms, we obtain

$$
\begin{aligned}
F_{\text {sing }}= & \frac{i}{q^{2}} \sum_{j=1}^{m_{\mathrm{f}}} \exp \left(-i \mathbf{q} \cdot \mathbf{O} \mathbf{A}_{j}\right) \frac{\mathbf{q} \cdot\left(\mathbf{A}_{j+1} \mathbf{A}_{j} \times \mathbf{A}_{j-1} \mathbf{A}_{j}\right)}{\left(\mathbf{q} \cdot \mathbf{A}_{j+1} \mathbf{A}_{j}\right)\left(\mathbf{q} \cdot \mathbf{A}_{j-1} \mathbf{A}_{j}\right)} \\
= & \frac{i}{q^{2}} \exp \left(-i \mathbf{q} \cdot \mathbf{O} \mathbf{A}_{1}\right) \\
& \times \sum_{j=1}^{m_{\mathrm{f}}} \exp \left(-i \mathbf{q} \cdot \mathbf{A}_{1} \mathbf{A}_{i}\right) \frac{\mathbf{q} \cdot\left(\mathbf{A}_{j+1} \mathbf{A}_{j} \times \mathbf{A}_{j-1} \mathbf{A}_{j}\right)}{\left(\mathbf{q} \cdot \mathbf{A}_{j+1} \mathbf{A}_{j}\right)\left(\mathbf{q} \cdot \mathbf{A}_{j-1} \mathbf{A}_{j}\right)} \\
= & \frac{i q_{\perp}}{q^{2}} \exp \left(-i \mathbf{q} \cdot \mathbf{O} \mathbf{A}_{1}\right) \\
& \times \sum_{j=1}^{m_{\mathrm{f}}} \exp \left(-i \mathbf{q}_{\|} \cdot \mathbf{A}_{1} \mathbf{A}_{j}\right) \frac{\mathbf{n} \cdot\left(\mathbf{A}_{j+1} \mathbf{A}_{j} \times \mathbf{A}_{j-1} \mathbf{A}_{j}\right)}{\left(\mathbf{q}_{\|} \cdot \mathbf{A}_{j+1} \mathbf{A}_{j}\right)\left(\mathbf{q}_{\|} \cdot \mathbf{A}_{j-1} \mathbf{A}_{j}\right)} \\
= & \frac{i q_{\perp}}{q^{2}} \exp \left(-i \mathbf{q} \cdot \mathbf{O} \mathbf{A}_{1}\right) H .
\end{aligned}
$$

We need to calculate $H_{0}=\lim _{\mathbf{q}_{\|} \rightarrow \mathbf{0}} H$. But we can recognize in $H$ the two-dimensional form factor of the polygon $A_{1} \ldots A_{n}$. Its limit for $\mathbf{q}_{\|} \rightarrow \mathbf{0}$ is the area of the polygon,

$$
S_{\text {face }}=\frac{1}{2} \mathbf{n} \cdot \sum_{j=1}^{m_{\mathrm{f}}-1} \sum_{k=j+1}^{m_{\mathrm{f}}-1} \mathbf{A}_{j} \mathbf{A}_{j+1} \times \mathbf{A}_{k} \mathbf{A}_{k+1},
$$

and we have 


$$
\begin{aligned}
& F_{\text {sing }}\left(\mathbf{q}_{\|}=\right.\mathbf{0})=\frac{i}{2 q_{\perp}} \exp \left(-i \mathbf{q} \cdot \mathbf{O} \mathbf{A}_{1}\right) \\
& \times\left(\mathbf{n} \cdot \sum_{j=1}^{m_{\mathrm{f}}-1} \sum_{k=j+1}^{m_{\mathrm{f}}-1} \mathbf{A}_{j} \mathbf{A}_{j+1} \times \mathbf{A}_{k} \mathbf{A}_{k+1}\right) .
\end{aligned}
$$

The following points must be noted:

(a) The locus of the second-kind singularity is a line normal to the face and intersecting the origin, leading to poles on a sphere of radius $q$.

(b) For large $q_{\perp}$ values, the second-kind singularity behaves like $q^{-1}$.

(c) The second-kind singularity is proportional to the face area.

(d) The treatment of this second singularity includes partially terms involved previously in the treatment of the first singularity. For the term affected by the nullity of only one scalar product of the denominator, the treatment of the first singularity must be done using only $C_{i}$ and $D_{j}$ and taking advantage of the fact that their limits are equal.

\subsection{Third-kind singularities: vanishing $q$}

When $\mathbf{q}$ tends to $\mathbf{0}$, all the denominators are vanishing at the same time. Recalling the integral expression of the form factor, we have

$$
F_{\text {plhd }}(\mathbf{q}=\mathbf{0})=\iiint 1 \mathrm{~d}^{3} v=V_{\text {plhd }},
$$

where $V_{\text {plhd }}$ is the volume of the polyhedron.

\subsection{Faces nesting}

For symmetric polyhedra, it frequently occurs that two faces are parallel. In these cases, the directions corresponding to the second singularity associated with each of the parallel faces are the same, leading to well marked oscillations of period $2 \pi / d$ superposed on the $q^{-1}$ term, $d$ being the diameter joining the two faces. Consideration of the form factor of the tetrahedron for which such parallelism does not occur will clearly show that these oscillations are not intrinsic to the secondkind singularity, but must be attributed to the nesting of the faces.

\section{Numerical implementation and examples}

\subsection{Python program}

A numerical program using formula (15) must account for the singularities. Owing to their power-law nature, the treatment of these singularities can be done numerically by calculating the form factor in the immediate vicinity of the singularities. We have implemented such an approach in a Python program. A major point is to build a function which is called with an array of $\mathbf{q}$ values. Using such a method, a typical time of some $10^{-5} \mathrm{~s}$ per $\mathbf{q}$ value is obtained on a desk top computer for a $q$ array of size greater than 1000. Our implementation needs only the apex coordinate array, $\mathbf{A}_{i}$, and the face circuit array, $v_{j, k}$. As already mentioned, the travelling direction of each circuit is of major importance when using formula (15). This determination may be quite tedious for non-trivial polyhedra, and therefore it is performed by the application using only one point defining the interior of a given face. The whole application which corresponds to a Python class is available under the name poly_scat_ funct.py at http://www.insp.jussieu.fr/-7-Logiciels-.html and as supporting information for this article.

Note that our analytical treatment compared to cutting methods has the advantage of avoiding the artificial introduction of ghost faces and ghost edges. These ghost faces and ghost edges in a numerical implementation necessitate the subtraction of two singular terms, leading to possible numerical imprecision.

\subsection{Illustrative examples}

In this section, we will study several examples: a tetrahedron, a truncated cube and a stellated cube. We will pay special attention to the singularities and we will assess numerically the $q$ domain for which their power-law behaviour is clearly distinguishable. We will use dimensionless variables $F_{\text {plhd }} / V_{\text {plhd }}$ and $q_{\text {r }}=q\left(V_{\text {plhd }}\right)^{1 / 3}$. For all the studied cases, the limit of $F / V$ for small $q$ values is 1 , as it should be.

5.2.1. Tetrahedron. Let us consider the regular tetrahedron. Fig. 4 represents the variation of the form factor with $q$ for various q directions. For $q_{\mathrm{r}}>20$, three power-law behaviours are clearly visible. A least-squares fit of the form factor in the direction normal to a face allows us to determine a prefactor equal to 1.8018 , to be compared with $S_{\text {face }} / V^{2 / 3}=\frac{1}{2}\left(3^{7 / 6}\right) \simeq$ 1.8014 as given by the analytical study performed in the

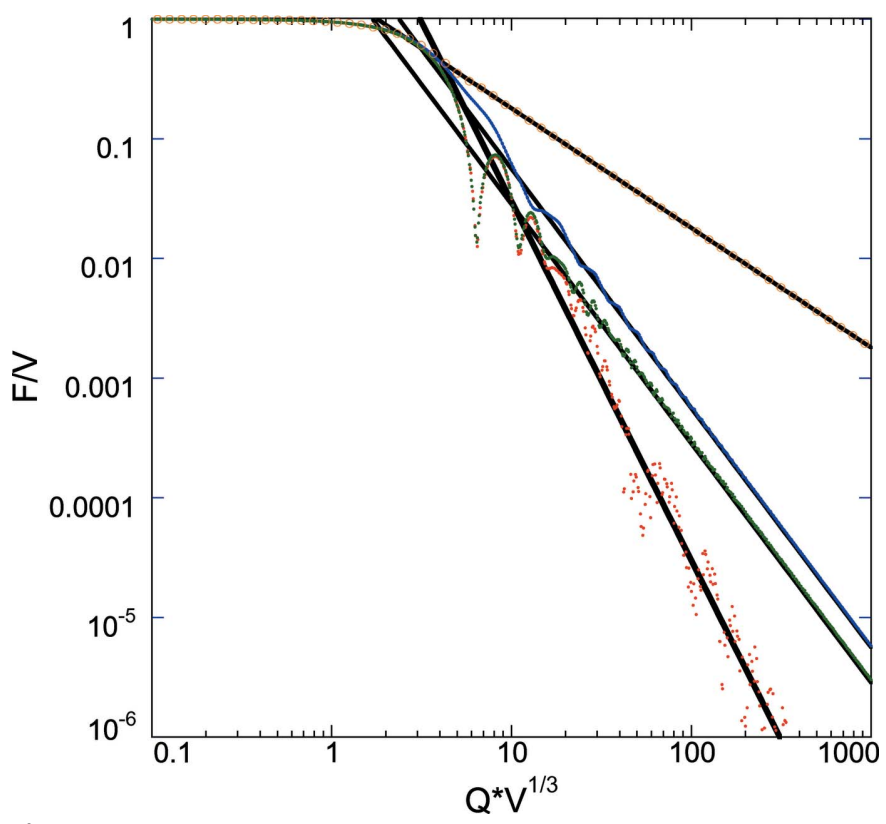

Figure 4

Form factor of a tetrahedron. Red dots: ordinary $\mathbf{q}$ direction $=(0.704$ 0.616 0.707). Blue dots: $\mathbf{q}$ direction perpendicular to an edge $=(100)$. Green dots: $\mathbf{q}$ direction perpendicular to an edge $=\left(1 / 2^{1 / 2} 01 / 2^{1 / 2}\right)$. Brown circles: $\mathbf{q}$ direction normal to a face $=(001)$. Full lines: best fits by $q^{-3}, q^{-2}$ and $q^{-1}$ laws. The multiplicative coefficient of the $q^{-1}$ law is 1.8018 , to be compared with $S_{\text {face }} / V^{2 / 3}=1.8014$. 
previous section. Note that in the direction normal to a face there is no oscillation of the intensity, while oscillations are clearly visible in the other directions. This is due to the absence of face nesting. This absence of nesting for the tetrahedron allows us to study the predicted presence of fringes parallel to the planes associated with the first-kind singularity. Fig. 5 shows the presence of such fringes: for large enough $q$ values $\left(q_{\mathrm{r}}>100\right)$ the fringes' period is in quite good accordance with the analytical prediction, $2 \pi / a$, and the fringes are already visible for $q_{\mathrm{r}}=30$. Fig. 6 represents a three-dimensional plot of the form factor for $q_{\mathrm{r}}=100$. The loci of the second singularities, poles, and of the first-kind singularities, great circles joining theses poles, are clearly visible.

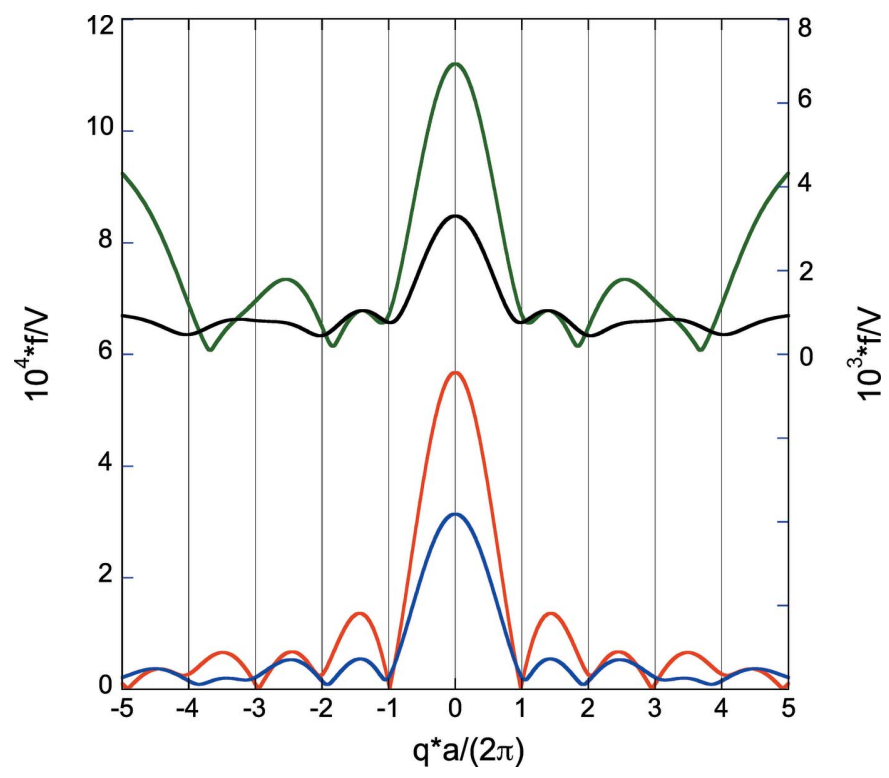

Figure 5

Cardinal sine fringes in the edge direction. Red line and left scale: $\mathbf{q}_{0}=$ $100 V^{1 / 3}(100)$. Blue line and left scale: $\mathbf{q}_{0}=100 V^{1 / 3}\left(1 / 2^{1 / 2} 01 / 2^{1 / 2}\right)$. Green line and right scale: $\mathbf{q}_{0}=30 V^{1 / 3}(100)$. Black line and right scale: $\mathbf{q}_{0}=30 V^{1 / 3}\left(1 / 2^{1 / 2} 01 / 2^{1 / 2}\right)$.

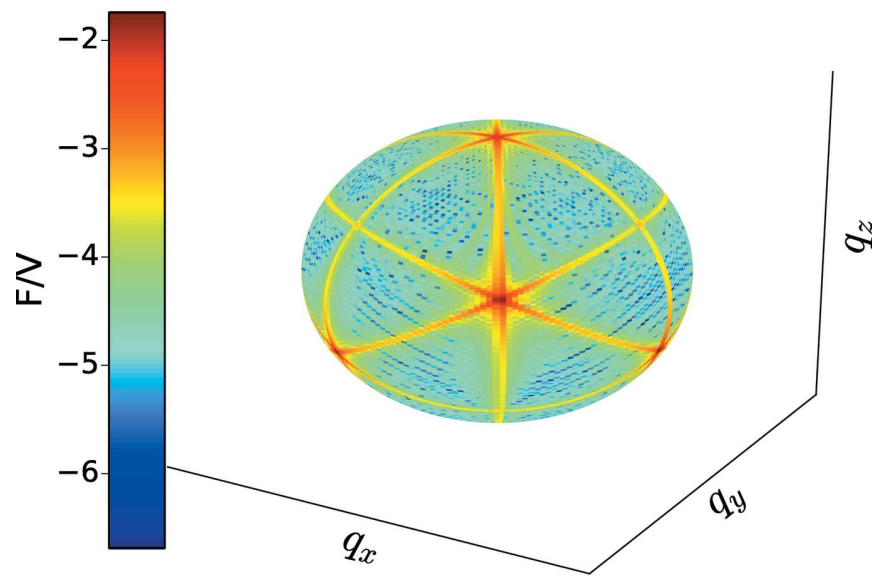

Figure 6

Three-dimensional plot of $F / V$ for a tetrahedron, $q V^{1 / 3}=100$. The colour scale is logarithmic.
5.2.2. Truncated cube. We will now study the well known case of the cube, but we will discuss the case of a cube truncated by chamfers along its edges, represented in Fig. 7. Such a truncated cube has three kinds of faces $-\{001\}$ large faces of area $S_{1}=\exp (2)(1-2 \varepsilon)^{2}, \quad\{011\}$ medium faces of area $S_{\mathrm{m}}=\exp (2)(1-2 \varepsilon)\left(2^{1 / 2} \varepsilon\right)$ and $\{111\}$ small faces of area $S_{\mathrm{s}}=\exp (2)\left(3^{1 / 2} / 2 \varepsilon^{2}\right)-$ and two types of edges - long edges of direction $\langle 100\rangle$ and short edges of direction $\langle 110\rangle$. Its volume is $V=\exp (3)\left(1-6 \varepsilon^{2}+16 / 3 \varepsilon^{3}\right)$. In Fig. 8 , the $q^{-1}$ power-law behaviour of $F / V$ is clearly visible for $q_{\mathrm{r}}>100$ for the directions normal to a face, while for directions perpendicular to an edge, the $q^{-2}$ power-law behaviour appears for $q_{\mathrm{r}}>1000$. Fig. 9 represents a three-dimensional plot of the form factor for $q_{\mathrm{r}}=200$. For the second-kind singularities, the singularities associated with the faces $\{001\}$ and $\{011\}$ are clearly visible, while a wide maximum appears in the direction $\{111\}$ corresponding to the small faces. For the first-kind singularities, the
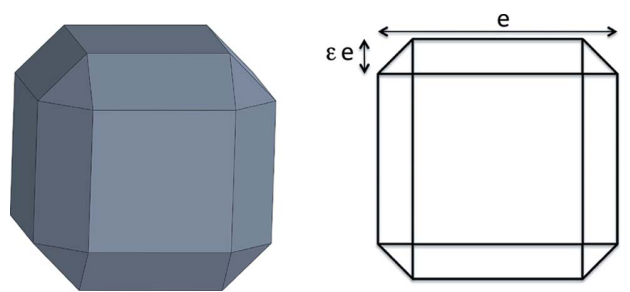

Figure 7

Schematic views of an edge-truncated cube.

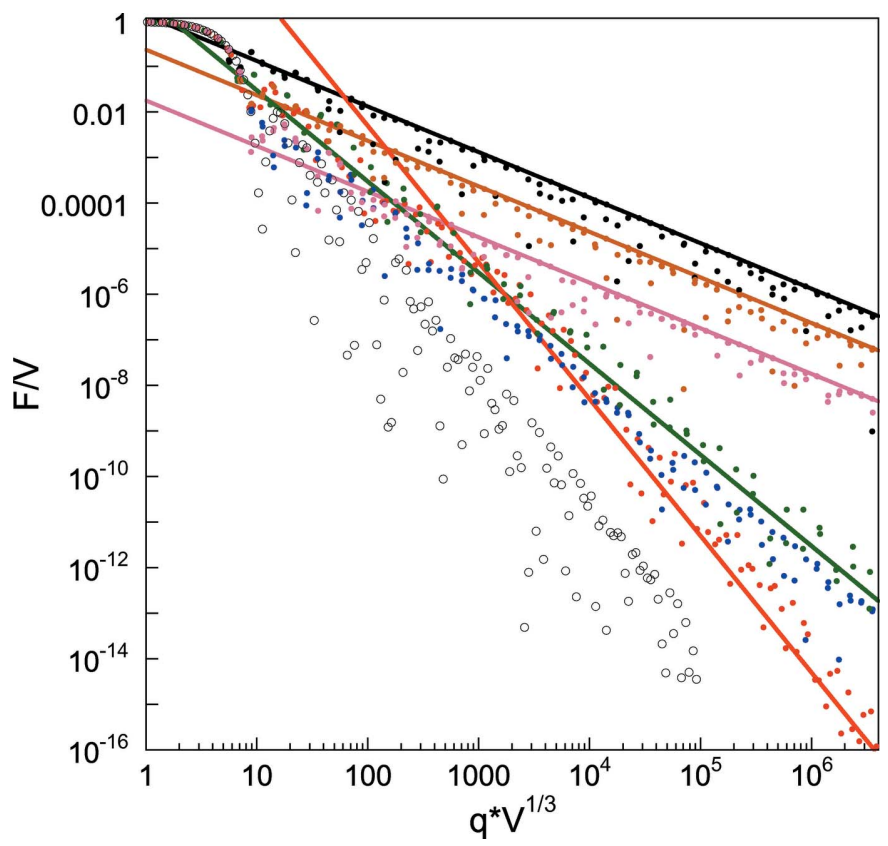

Figure 8

Form factor of an edge-truncated cube with $\varepsilon=10 \%$. Red dots: ordinary $\mathbf{q}$ direction $=\left(\begin{array}{ll}0.704 & 0.6160 .707\end{array}\right)$; red line: $5000\left(q V^{1 / 3}\right)^{-3}$. Blue dots: $\mathbf{q}$ direction perpendicular to a short edge $=\left(1 / 2^{1 / 2} 1 / 21 / 2\right)$. Green dots: $\mathbf{q}$ direction perpendicular to a long edge $=\left(3^{1 / 2} / 21 / 20\right)$; green line: $3\left(q V^{1 / 3}\right)^{-2}$. Black dots: $\mathbf{q}$ direction normal to a large face $=(001)$; black line: $2 S_{1}\left(q V^{1 / 3}\right)^{-1}$. Brown dots: $\mathbf{q}$ direction normal to a medium face $=$ $\left(1 / 2^{1 / 2} 1 / 2^{1 / 2} 0\right)$; brown line: $2 S_{\mathrm{m}}\left(q V^{1 / 3}\right)^{-1}$. Pink dots: $\mathbf{q}$ direction normal to a small face $=\left(1 / 3^{1 / 2} 1 / 3^{1 / 2} 1 / 3^{1 / 2}\right)$; pink line: $2 S_{\mathrm{s}}\left(q V^{1 / 3}\right)^{-1}$. Black open circles: $\mathbf{q}$ direction $=\left(1 / 3^{1 / 2} 1 / 3^{1 / 2} 1 / 3^{1 / 2}\right)$, for an untruncated cube. 


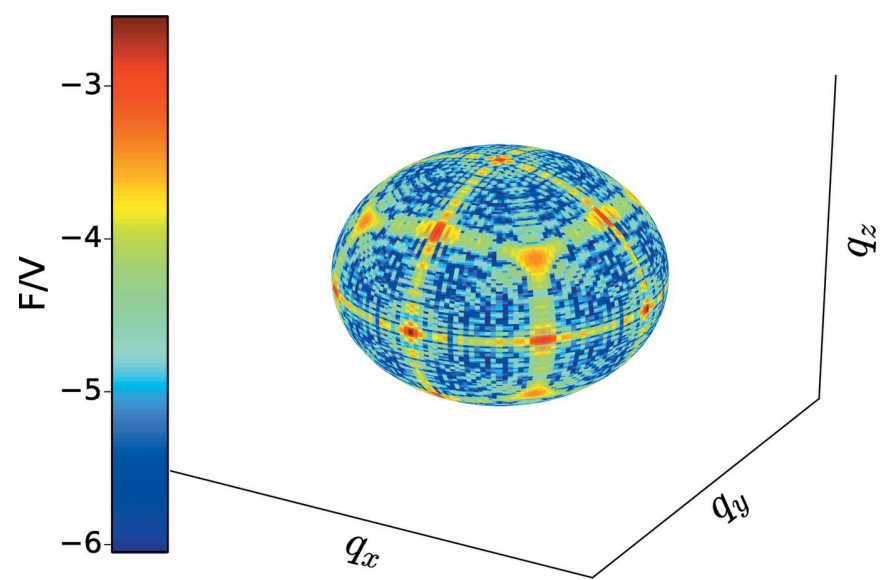

Figure 9

Three-dimensional plot of $F / V$ for a truncated cube, $q V^{1 / 3}=200$. The colour scale is logarithmic.
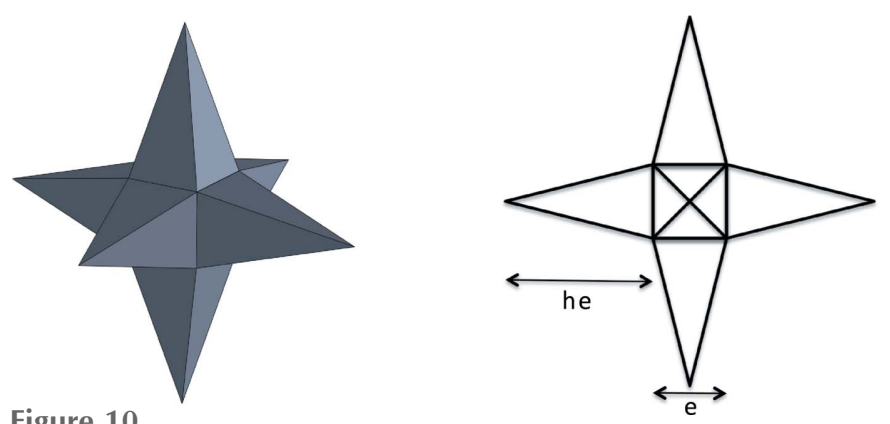

Figure 10

Schematic views of a stellated cube.

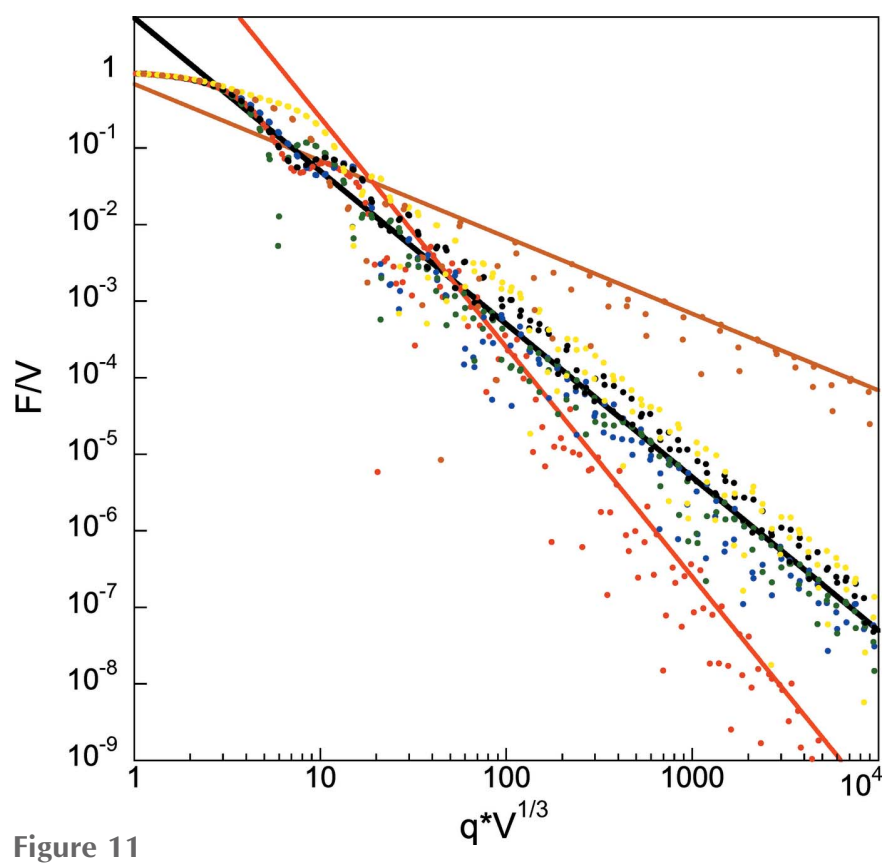

Form factor of a stellated cube $e=1, h=2$. Red dots: ordinary $\mathbf{q}$ direction $=(0.7040 .6160 .707)$; red line: $250\left(q V^{1 / 3}\right)^{-3}$. Blue dots: q direction perpendicular to a 'base' edge $=\left(3^{1 / 2} / 21 / 20\right)$. Green dots: q direction perpendicular to a 'tip' edge $=(2 / 32 / 31 / 3)$. Black dots: $\mathbf{q}$ direction normal to both a 'base' edge and a 'tip' 'edge' = $\left(1 / 2^{1 / 2} 1 / 2^{1 / 2} 0\right)$; black line: $5\left(q V^{1 / 3}\right)^{-2}$. Yellow dots: q direction normal to four 'base' edges $=(001)$. Brown dots: $\mathbf{q}$ direction normal to a face $=$ $(01 / 2 h) /\left(h^{2}+1 / 4\right)^{1 / 2}$; brown line: $2 S\left(q V^{1 / 3}\right)^{-1}$.

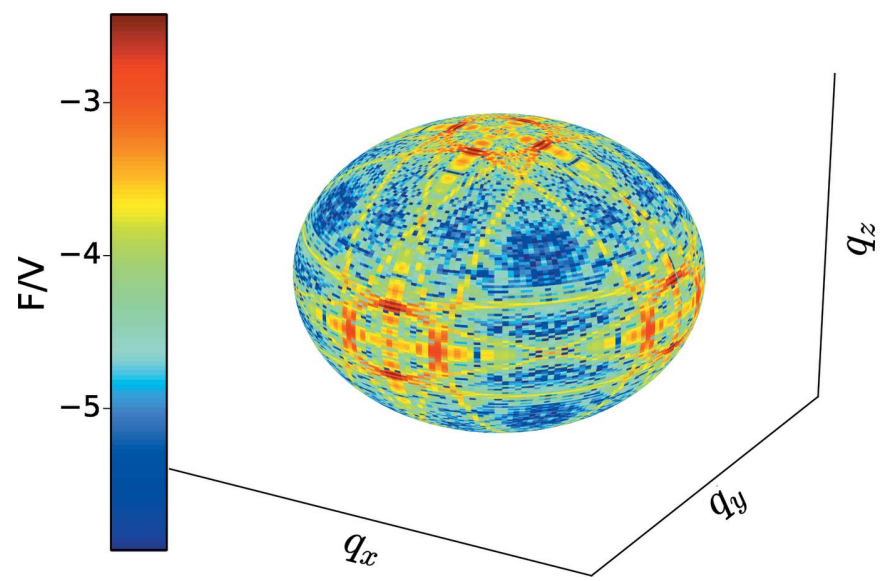

Figure 12

Three-dimensional plot of $F / V$ for a stellated cube, $q V^{1 / 3}=150$. The colour scale is logarithmic.

great circles associated with the edges $\langle 100\rangle$ are clearly visible, while the great circles associated with the edges $\langle 110\rangle$ are wide. Because of the nesting, oscillations are observed along every singular direction.

5.2.3. Stellated cube. Let us consider the case of a stellated cube, represented in Fig. 10. Such a stellated cube has one kind of face, $\{01 / 2 h\}$, and two types of edge, the 'base' edges of direction $\langle 100\rangle$ and the 'tip' edges of direction $\langle h 1 / 21 / 2\rangle$. Its volume is $V=\exp (3)(1+2 h)$. In Fig. 11, the $q^{-1}$ power-law behaviour of $F / V$ is clearly visible for $q_{\mathrm{r}}>20$ for the direction normal to a face, while for directions perpendicular to an edge, the $q^{-2}$ power-law behaviour appears for $q_{\mathrm{r}}>100$. Note that the direction (001), which corresponds to a first-kind singularity, behaves effectively as $q^{-2}$, whereas this direction would correspond to a ghost face in a naive cutting procedure. Fig. 12 represents a three-dimensional plot of the form factor for $q_{\mathrm{r}}=150$. The poles associated with the normals to the faces are clearly visible, together with both the great circles associated with the edges $\langle 100\rangle$ and the great circles associated with the edges $\langle h 1 / 21 / 2\rangle$. Because of the nesting, oscillations are observed along every singular direction.

\section{Conclusion}

The use of vectorial notations and a careful examination of the summation procedure involved in the calculation of the form factor of a polyhedron, $F_{\text {plhd }}$, allows us to obtain a general and compact formula using only the apex coordinates and the connection circuit of each apex. The formula is valid for a very large class of polyhedra, the polyhedra severable into convex polyhedra.

A first advantage of this formula is to allow a generic analysis of the singularities. Three classes of $\mathbf{q}$ directions appear: the ordinary direction for which $F_{\text {plhd }}$ behaves like $q^{-3}$ for large $q$, the directions perpendicular to an edge for which a $q^{-2}$ behaviour is found and the directions normal to a face for which a $q^{-1}$ behaviour is found. These singularities are intrinsically associated with the presence of truncations in the polyhedron. Their intensities are proportional, respectively, to 
the edge lengths and the face areas. The $q$ range for which they are easily distinguishable depends also on the geometrical details of the system. The numerical study that we have performed on some examples seems to indicate that observation of the singularities is easier for 'acute' polyhedra (a tetrahedron and a stellated cube) than for 'smooth' polyhedra (a truncated cube). As shown by the comparison of the case of the tetrahedron with the case of the truncated cube, the nesting of faces leads to the appearance of oscillations concomitant with the singularities.

A second advantage of this formula is to avoid the need to cut complex polyhedra into elementary parts. The construction of a form factor catalogue is therefore useless.

We think that the generality of the formula that we have obtained will facilitate the structural determination of superlattices. In the field of coherent scattering by single particles, which will benefit from the development of high-quality facilities like low-emittance synchrotrons and X-ray freeelectron lasers, the general results that we have established on singularities may allow an optimization of the data acquisition strategy.

\section{APPENDIX $A$}

\section{Inductive demonstration of formula (4)}

We have established by direct calculation the formula for a triangle. With the hypothesis that the formula is correct for a $p$-gon, we will show that the formula is valid for a $(p+1)$-gon. Adding a supplementary side between the apices $i+1$ and $i+3$, we cut the $(p+1)$-gon in two parts: a $p$-gon and a triangle, as shown in Fig. 13. We can calculate the form factor of the $(p+1)$-gon using formula (4) applied to those two parts and using the additivity of the integration. In this sum, the coefficient of the phase term associated with the apex $i+1$, $c$, is

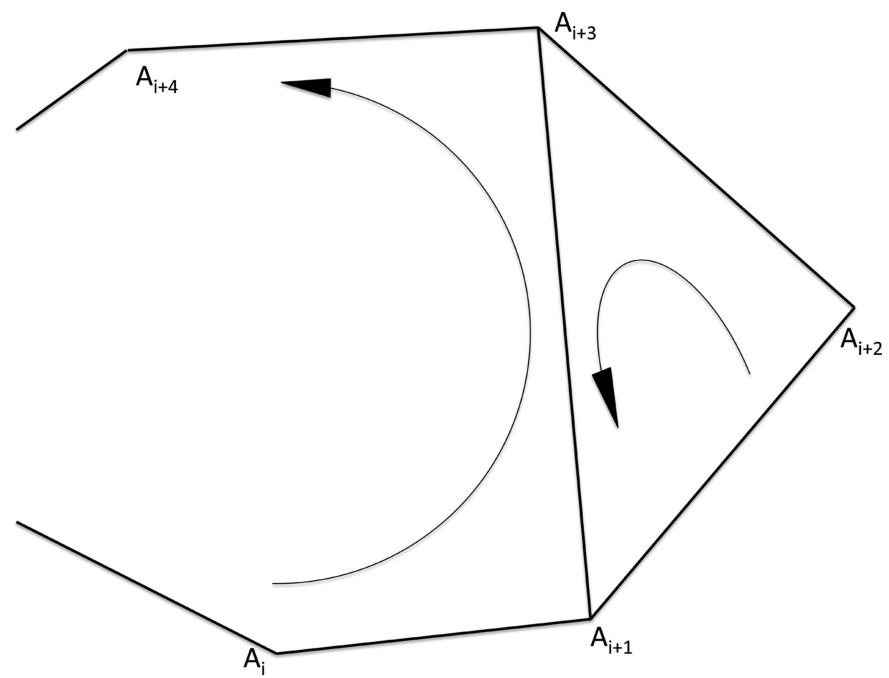

Figure 13

Cutting of a $(p+1)$-gon into a $p$-gon and a triangle. Note the difference in circuit directions for the edge $A_{i+1} A_{i+3}$ for the triangle and the $p$-gon.

$$
\begin{aligned}
& c=\mathbf{n} \cdot\left[\frac{\mathbf{A}_{i+1} \mathbf{A}_{i+3} \times \mathbf{A}_{i} \mathbf{A}_{i+1}}{\left(\mathbf{q} \cdot \mathbf{A}_{i} \mathbf{A}_{i+1}\right)\left(\mathbf{q} \cdot \mathbf{A}_{i+1} \mathbf{A}_{i+3}\right)}\right. \\
& \left.+\frac{\mathbf{A}_{i+1} \mathbf{A}_{i+2} \times \mathbf{A}_{i+3} \mathbf{A}_{i+1}}{\left(\mathbf{q} \cdot \mathbf{A}_{i+3} \mathbf{A}_{i+1}\right)\left(\mathbf{q} \cdot \mathbf{A}_{i+1} \mathbf{A}_{i+2}\right)}\right] \\
& =\frac{\mathbf{n}}{\left(\mathbf{q} \cdot \mathbf{A}_{i} \mathbf{A}_{i+1}\right)\left(\mathbf{q} \cdot \mathbf{A}_{i+1} \mathbf{A}_{i+2}\right)} \\
& \cdot\left[\frac{\left(\mathbf{q} \cdot \mathbf{A}_{i+1} \mathbf{A}_{i+2}\right)\left(\mathbf{A}_{i+1} \mathbf{A}_{i+3} \times \mathbf{A}_{i} \mathbf{A}_{i+1}\right)}{\left(\mathbf{q} \cdot \mathbf{A}_{i+1} \mathbf{A}_{i+3}\right)}\right. \\
& \left.+\frac{\left(\mathbf{q} \cdot \mathbf{A}_{i} \mathbf{A}_{i+1}\right)\left(\mathbf{A}_{i+1} \mathbf{A}_{i+2} \times \mathbf{A}_{i+1} \mathbf{A}_{i+3}\right)}{\left(\mathbf{q} \cdot \mathbf{A}_{i+1} \mathbf{A}_{i+3}\right)}\right] \\
& =\frac{\mathbf{n}}{\left(\mathbf{q} \cdot \mathbf{A}_{i} \mathbf{A}_{i+1}\right)\left(\mathbf{q} \cdot \mathbf{A}_{i+1} \mathbf{A}_{i+2}\right)} \\
& \cdot\left[\frac{-\left(\mathbf{q} \cdot \mathbf{A}_{i+1} \mathbf{A}_{i+2}\right) \mathbf{A}_{i} \mathbf{A}_{i+1}+\left(\mathbf{q} \cdot \mathbf{A}_{i} \mathbf{A}_{i+1}\right) \mathbf{A}_{i+1} \mathbf{A}_{i+2}}{\left(\mathbf{q} \cdot \mathbf{A}_{i+1} \mathbf{A}_{i+3}\right)}\right. \\
& \left.\times \mathbf{A}_{i+1} \mathbf{A}_{i+3}\right] \\
& =\frac{\mathbf{n}}{\left(\mathbf{q} \cdot \mathbf{A}_{i} \mathbf{A}_{i+1}\right)\left(\mathbf{q} \cdot \mathbf{A}_{i+1} \mathbf{A}_{i+2}\right)} \\
& \cdot\left[\frac{\mathbf{q} \times\left(\mathbf{A}_{i+1} \mathbf{A}_{i+2} \times \mathbf{A}_{i} \mathbf{A}_{i+1}\right)}{\left(\mathbf{q} \cdot \mathbf{A}_{i+1} \mathbf{A}_{i+3}\right)} \times \mathbf{A}_{i+1} \mathbf{A}_{i+3}\right] \\
& =\frac{\mathbf{n}}{\left(\mathbf{q} \cdot \mathbf{A}_{i} \mathbf{A}_{i+1}\right)\left(\mathbf{q} \cdot \mathbf{A}_{i+1} \mathbf{A}_{i+2}\right)} \\
& \cdot\left\{\frac{\left(\mathbf{q} \cdot \mathbf{A}_{i+1} \mathbf{A}_{i+3}\right)\left(\mathbf{A}_{i+1} \mathbf{A}_{i+2} \times \mathbf{A}_{i} \mathbf{A}_{i+1}\right)}{\left(\mathbf{q} \cdot \mathbf{A}_{i+1} \mathbf{A}_{i+3}\right)}\right. \\
& \left.-\frac{\left[\mathbf{A}_{i+1} \mathbf{A}_{i+3} \cdot\left(\mathbf{A}_{i+1} \mathbf{A}_{i+2} \times \mathbf{A}_{i} \mathbf{A}_{i+1}\right)\right] \mathbf{q}}{\left(\mathbf{q} \cdot \mathbf{A}_{i+1} \mathbf{A}_{i+3}\right)}\right\} .
\end{aligned}
$$

Since all the apices of the $(p+1)$-gon are coplanar, we have $\left[\mathbf{A}_{i+1} \mathbf{A}_{i+3} \cdot\left(\mathbf{A}_{i+1} \mathbf{A}_{i+2} \times \mathbf{A}_{i} \mathbf{A}_{i+1}\right)\right]=0$ and we ultimately find

$$
c=\frac{\mathbf{n} \cdot\left(\mathbf{A}_{i+1} \mathbf{A}_{i+2} \times \mathbf{A}_{i} \mathbf{A}_{i+1}\right)}{\left(\mathbf{q} \cdot \mathbf{A}_{i} \mathbf{A}_{i+1}\right)\left(\mathbf{q} \cdot \mathbf{A}_{i+1} \mathbf{A}_{i+2}\right)},
$$

which is the coefficient we need for the phase term associated with the apex $i+1$ in the application of formula (4) to the $(p+1)$-gon. The same calculation can be performed for the phase term associated with the apex $i+3$. The coefficient of the phase term associated with the apex $i+2$ is the same for the $(p+1)$-gon and the triangle, and the coefficients associated with all the other apices are the same for the $(p+1)$ gon and the $p$-gon. All together, these results demonstrate the validity of formula (4) for the $(p+1)$-gon.

\section{APPENDIX $B$}

Vanishing of the coefficient of the phase term $\exp (-i \mathrm{q} \cdot \mathrm{OM})$

We want to demonstrate the vanishing of the coefficient

$$
G_{0}=\sum_{i=1}^{n_{\mathrm{a}}}\left[\sum_{s=1}^{p(i)} \frac{\mathbf{M} \mathbf{A}_{i} \cdot\left(\mathbf{A}_{w_{i, s}} \mathbf{A}_{i} \times \mathbf{A}_{i} \mathbf{A}_{w_{i, s-1}}\right)}{\left(\mathbf{q} \cdot \mathbf{A}_{w_{i, s}} \mathbf{A}_{i}\right)\left(\mathbf{q} \cdot \mathbf{A}_{i} \mathbf{A}_{w_{i, s-1}}\right)\left(\mathbf{q} \cdot \mathbf{M} \mathbf{A}_{i}\right)}\right] .
$$

Using the expression of each of its components established when calculating the coefficients of the other phase terms, we have 


$$
G_{0}=\sum_{i=1}^{n_{\mathrm{a}}}\left[\sum_{s=1}^{p(i)} \frac{\mathbf{q} \cdot\left(\mathbf{A}_{w_{i, s}} \mathbf{A}_{i} \times \mathbf{A}_{w_{i, s-1}} \mathbf{A}_{i}\right)}{\left(\mathbf{q} \cdot \mathbf{A}_{w_{i, s}} \mathbf{A}_{i}\right)\left(\mathbf{q} \cdot \mathbf{A}_{w_{i, s-1}} \mathbf{A}_{i}\right) q^{2}}\right] .
$$

Using the indexings by the circuit associated with the faces, we have

$$
G_{0}=\sum_{j=1}^{n_{\mathrm{f}}}\left[\sum_{k=1}^{m(j)} \frac{\mathbf{q} \cdot\left(\mathbf{A}_{v_{j, k+1}} \mathbf{A}_{v_{j, k}} \times \mathbf{A}_{v_{j, k-1}} \mathbf{A}_{v_{j, k}}\right)}{\left(\mathbf{q} \cdot \mathbf{A}_{v_{j, k+1}} \mathbf{A}_{v_{j, k}}\right)\left(\mathbf{q} \cdot \mathbf{A}_{v_{j, k-1}} \mathbf{A}_{v_{j, k}}\right) q^{2}}\right] .
$$

For a triangular face from which the apices are labelled $A_{1}, A_{2}$, $A_{3}$ we have

$$
\begin{aligned}
\sum_{k=1}^{m(j)} & \frac{\mathbf{q} \cdot\left(\mathbf{A}_{v_{j, k+1}} \mathbf{A}_{v_{j, k}} \times \mathbf{A}_{v_{j, k-1}} \mathbf{A}_{v_{j, k}}\right)}{\left(\mathbf{q} \cdot \mathbf{A}_{v_{j, k+1}} \mathbf{A}_{v_{j, k}}\right)\left(\mathbf{q} \cdot \mathbf{A}_{v_{j, k-1}} \mathbf{A}_{v_{j, k}}\right) q^{2}} \\
= & \frac{\mathbf{q} \cdot\left(\mathbf{A}_{2} \mathbf{A}_{1} \times \mathbf{A}_{3} \mathbf{A}_{1}\right)}{\left(\mathbf{q} \cdot \mathbf{A}_{2} \mathbf{A}_{1}\right)\left(\mathbf{q} \cdot \mathbf{A}_{3} \mathbf{A}_{1}\right) q^{2}} \\
& +\frac{\mathbf{q} \cdot\left(\mathbf{A}_{3} \mathbf{A}_{2} \times \mathbf{A}_{1} \mathbf{A}_{2}\right)}{\left(\mathbf{q} \cdot \mathbf{A}_{3} \mathbf{A}_{2}\right)\left(\mathbf{q} \cdot \mathbf{A}_{1} \mathbf{A}_{2}\right) q^{2}} \\
& +\frac{\mathbf{q} \cdot\left(\mathbf{A}_{1} \mathbf{A}_{3} \times \mathbf{A}_{2} \mathbf{A}_{3}\right)}{\left(\mathbf{q} \cdot \mathbf{A}_{1} \mathbf{A}_{3}\right)\left(\mathbf{q} \cdot \mathbf{A}_{2} \mathbf{A}_{3}\right) q^{2}}
\end{aligned}
$$

Using the closure relation $\mathbf{A}_{1} \mathbf{A}_{3}+\mathbf{A}_{3} \mathbf{A}_{2}+\mathbf{A}_{2} \mathbf{A}_{1}=\mathbf{0}$, we have

$$
\mathbf{A}_{3} \mathbf{A}_{2} \times \mathbf{A}_{1} \mathbf{A}_{2}=\mathbf{A}_{3} \mathbf{A}_{1} \times \mathbf{A}_{1} \mathbf{A}_{2}=\mathbf{A}_{2} \mathbf{A}_{1} \times \mathbf{A}_{3} \mathbf{A}_{1}
$$

and

$$
\mathbf{A}_{1} \mathbf{A}_{3} \times \mathbf{A}_{2} \mathbf{A}_{3}=\mathbf{A}_{1} \mathbf{A}_{3} \times \mathbf{A}_{2} \mathbf{A}_{1}=\mathbf{A}_{2} \mathbf{A}_{1} \times \mathbf{A}_{3} \mathbf{A}_{1} .
$$

The three terms have the same numerator. Factorization leads to

$$
\begin{aligned}
\frac{1}{\left(\mathbf{q} \cdot \mathbf{A}_{2} \mathbf{A}_{1}\right)\left(\mathbf{q} \cdot \mathbf{A}_{3} \mathbf{A}_{1}\right)} & +\frac{1}{\left(\mathbf{q} \cdot \mathbf{A}_{3} \mathbf{A}_{2}\right)\left(\mathbf{q} \cdot \mathbf{A}_{1} \mathbf{A}_{2}\right)} \\
& +\frac{1}{\left(\mathbf{q} \cdot \mathbf{A}_{1} \mathbf{A}_{3}\right)\left(\mathbf{q} \cdot \mathbf{A}_{2} \mathbf{A}_{3}\right)}
\end{aligned}
$$

and finally to

$$
\frac{\mathbf{q} \cdot \mathbf{A}_{3} \mathbf{A}_{2}-\mathbf{q} \cdot \mathbf{A}_{3} \mathbf{A}_{1}+\mathbf{q} \cdot \mathbf{A}_{2} \mathbf{A}_{1}}{\left(\mathbf{q} \cdot \mathbf{A}_{2} \mathbf{A}_{1}\right)\left(\mathbf{q} \cdot \mathbf{A}_{3} \mathbf{A}_{1}\right)\left(\mathbf{q} \cdot \mathbf{A}_{3} \mathbf{A}_{2}\right)}=0 .
$$

Using the same inductive method as used in Appendix $A$, the vanishing of the coefficient associated with a triangular face can be generalized to a face with $p$ apices. Each partial sum involved in the general sum, $G_{0}$, is therefore null, leading to the nullity of $G_{0}$.

\section{APPENDIX C}

\section{Generalization to branched polyhedra: some cutting} tools

\section{C1. Lemma 1: Adding a 'coplanar' edge}

Consider a circuit of edges around an apex, $\mathbf{V}_{1} \ldots \mathbf{V}_{n}$. If we add artificially between the edge $\mathbf{V}_{i}$ and the edge $\mathbf{V}_{i+1}$ an edge $\mathbf{W}$ coplanar with them, the part of the new sum around the apex involving $\mathbf{W}$ will be

$$
R=\frac{\left(\mathbf{q}, \mathbf{V}_{i+1}, \mathbf{W}\right)}{\left(\mathbf{q} \cdot \mathbf{V}_{i+1}\right)(\mathbf{q} \cdot \mathbf{W}) q^{2}}+\frac{\left(\mathbf{q}, \mathbf{W}, \mathbf{V}_{i}\right)}{(\mathbf{q} \cdot \mathbf{W})\left(\mathbf{q} \cdot \mathbf{V}_{i}\right) q^{2}}
$$

The three vectors being coplanar, we have $\mathbf{W}=\xi \mathbf{V}_{i+1}+\zeta \mathbf{V}_{i}$, which leads to

$$
\begin{aligned}
R= & \frac{\zeta\left(\mathbf{q}, \mathbf{V}_{i+1}, \mathbf{V}_{i}\right)}{\left(\mathbf{q} \cdot \mathbf{V}_{i+1}\right)\left[\xi\left(\mathbf{q} \cdot \mathbf{V}_{i+1}\right)+\zeta\left(\mathbf{q} \cdot \mathbf{V}_{i}\right)\right] q^{2}} \\
& +\frac{\xi\left(\mathbf{q}, \mathbf{V}_{i+1}, \mathbf{V}_{i}\right)}{\left(\mathbf{q} \cdot \mathbf{V}_{i}\right)\left[\xi\left(\mathbf{q} \cdot \mathbf{V}_{i+1}\right)+\zeta\left(\mathbf{q} \cdot \mathbf{V}_{i}\right)\right] q^{2}} \\
= & \frac{\left[\xi\left(\mathbf{q} \cdot \mathbf{V}_{i+1}\right)+\zeta\left(\mathbf{q} \cdot \mathbf{V}_{i}\right)\right]\left(\mathbf{q}, \mathbf{V}_{i+1}, \mathbf{V}_{i}\right)}{\left(\mathbf{q} \cdot \mathbf{V}_{i}\right)\left(\mathbf{q} \cdot \mathbf{V}_{i+1}\right)\left[\xi\left(\mathbf{q} \cdot \mathbf{V}_{i+1}\right)+\zeta\left(\mathbf{q} \cdot \mathbf{V}_{i}\right)\right] q^{2}} \\
= & \frac{\left(\mathbf{q}, \mathbf{V}_{i+1}, \mathbf{V}_{i}\right)}{\left(\mathbf{q} \cdot \mathbf{V}_{i}\right)\left(\mathbf{q} \cdot \mathbf{V}_{i+1}\right) q^{2}}
\end{aligned}
$$

This is just the term involving $\mathbf{V}_{i+1}$ and $\mathbf{V}_{i}$ in the old sum before adding $\mathbf{W}$.

\section{C2. Lemma 2: Adding an apex in the middle of a face}

Let us add artificially an apex in the middle of a face. Using lemma 1, this addition does not change the circuit sum around the former apices. For the new apex, the use of lemma 1 allows us to suppress one after the other all the new edges until we have only two of them, for which the circuit sum is obviously null, the predecessor of one of the edges being also its successor. It is worth remarking that this addition of an apex can be done on the periphery of the face, i.e. on an edge, since, in every circuit sum, only the directions of the edges are relevant.

\section{C3. Lemma 3: joining two polyhedra by a face}

A polyhedron may be built by joining by a face two polyhedra. For this face, the interior of the first polyhedron is the exterior of the other one and vice versa. Therefore, when the summation is performed using the indexing of the apices by faces the term associated with this face disappears. In the same way, a polyhedron may be obtained by digging a hole from a face. Once again, the terms associated with the face from which the hole is dug disappear in the expression of the form factor.

\section{C4. Branched polyhedra severable into convex polyhedra}

By using the three previous lemmas, formula (15) established for not too concave polyhedra can be generalized to every polyhedron severable into convex polyhedra. While the precise topological study of polyhedra is far outside the scope of this paper, we believe that this generalization is valid in practice for most of the useful polyhedra.

\section{Acknowledgements}

We thank Jean-Noël Aqua, Romain Bernard, Léo BossardGiannesini and Geoffroy Prévot for fruitful discussions and William Sacks for careful reading.

\section{References}

Babonneau, D. (2010). J. Appl. Cryst. 43, 929-936.

Balmes, O., Prevot, G., Torrelles, X., Lundgren, E. \& Ferrer, S. (2016). ACS Catal. 6, 1285-1291. 
Cho, K.-S., Talapin, D. V., Gaschler, W. \& Murray, C. B. (2005). J. Am. Chem. Soc. 127, 7140-7147.

Chourou, S. T., Sarje, A., Li, X. S., Chan, E. R. \& Hexemer, A. (2013). J. Appl. Cryst. 46, 1781-1795.

Georg, K. \& Tausch, J. (1994). Math. C, 62, 755-763.

Geuchies, J. J. et al. (2016). Nat. Mater. 15, 1248-1254.

Goubet, N., Albouy, P.-A., Thompson, A. \& Pileni, M.-P. (2016). CrystEngComm, 18, 6166-6175.

Goubet, N., Yan, C., Polli, D., Portalès, H., Arfaoui, I., Cerullo, G. \& Pileni, M.-P. (2013). Nano Lett. 13, 504-508.

Gruner, M. E. G., Rollmann, G., Entel, P. \& Farle, M. (2008). Phys. Rev. Lett. 100, 087203.

Jones, M. R., Macfarlane, R. J., Lee, B., Zhang, J., Young, K. L., Senesi, A. J. \& Mirkin, C. A. (2010). Nat. Mater. 9, 913-917.

Kalesaki, E., Delerue, C., Morais Smith, C., Beugeling, W., Allan, G. \& Vanmaekelbergh, D. (2014). Phys. Rev. X, 4, 011010.

Langille, M. R., Personick, M. L., Zhang, J. \& Mirkin, C. A. (2012). J. Am. Chem. Soc. 134, 14542-14554.
Lazzari, R. (2002). J. Appl. Cryst. 35, 406-421.

Lee, J.-S., Kovalenko, M. V., Huang, J., Chung, D. S. \& Talapin, D. V. (2011). Nat. Nanotechnol. 6, 348-352.

Murphy, C. J., Sau, T. K., Gole, A. M., Orendorff, C. J., Gao, J., Gou, L., Hunyadi, S. E. \& Li, T. (2005). J. Phys. Chem. B, 109, 1385713870.

Renaud, G., Lazzari, R. \& Leroy, F. (2009). Surf. Sci. Rep. 64, 255380.

Senesi, A. \& Lee, B. (2015). J. Appl. Cryst. 48, 565-577.

Xia, Y., Xiong, Y., Lim, B. \& Skrabalak, S. E. (2009). Angew. Chem. Int. Ed. 48, 60-103.

Xie, S., Lu, N., Xie, Z., Wang, J., Kim, M. J. \& Xia, Y. (2012). Angew. Chem. Int. Ed. 51, 10266-10270.

Yamada, Y., Tsung, C.-K., Huang, W., Huo, Z., Habas, S. E., Soejima, T., Aliaga, C. E., Somorjai, G. A. \& Yang, P. (2011). Nat. Chem. 3, 372-376.

Zhang, H., Jin, M. \& Xia, Y. (2012). Angew. Chem. Int. Ed. 51, 76567673 . 